\title{
Costos de Ajuste del Capital y Dinámica de las Firmas: \\ Shocks y Efectos sobre la Demanda de Factores *
}

\author{
Germán Bet ${ }^{* *}$ \\ Tesis \\ Maestría en Economía \\ Universidad Nacional de La Plata
}

Directores:

Irene Brambilla

Guido Porto

10 de Mayo de 2011

\footnotetext{
*Agradezco especialmente a mis directores Irene Brambilla y Guido Porto por su colaboración y dedicación durante el proceso de tesis. Agradezco también a Marcelo Oviedo, a Cecilia Peluffo, y a los participantes del Seminario de Tesis por sus comentarios y sugerencias. Errores y/u omisiones son de mi exclusiva responsabilidad.

** Departamento de Economía, Universidad Nacional de La Plata, y CEDLAS (Centro de Estudios Distributivos, Laborales y Sociales. Facultad de Ciencias Económicas. Universidad Nacional de La Plata).E-mail: betgerman@gmail.com
} 


\title{
Resumen
}

El presente trabajo analiza la estructura de los costos de ajuste del capital para el caso de la industria manufacturera Argentina. Haciendo uso de un modelo estructural de demanda por inversión intertemporal de la firma, se estiman los costos de ajuste del capital para un panel de plantas manufactureras argentinas. Los parámetros estimados son utilizados luego en experimentos contrafácticos con el objetivo de evaluar los efectos de shocks sobre la demanda de factores. Los resultados obtenidos sugieren la existencia de convexidades, no-convexidades e irreversibilidades en la forma funcional de los costos de ajuste. En particular, se estiman altos costos fijos y de transacción. Asimismo, los ejercicios de simulación contrafácticos resaltan la importancia de considerar la movilidad del capital y sus fricciones a fines de evaluar los efectos de shocks sobre los mercados de factores. Tanto la velocidad como la magnitud del ajuste de los mercados dependen directamente de la movilidad del capital y de los costos de ajuste de este.

Códigos JEL: E22, E24, J2, C15.

Palabras Claves: Costos de Ajuste del Capital, Inversión, Dinámica del Mercado Laboral, Método de Momentos Simulados.

\begin{abstract}
This paper studies the nature of capital adjustment at the plant-level for the manufacturing industry of Argentina. We use a dynamic structural model of capital accumulation in order to estimate the capital adjustment costs parameters. We fit this model to plant-level panel data from Argentina. We obtain a measure of firm's capital adjustment costs and use them in counterfactual simulations to assess the effects of profitability shocks on factor demands. Our findings indicate the existence of both convex and non-convex costs at the plant-level. Specifically, we estimate large fixed and transaction costs. Additionally, counterfactual simulations highlight the importance of modeling capital mobility and its frictions. The speed of adjustment of the market as well as the magnitude of the impact of the shock on factor demands depend on capital mobility and its adjustment costs.
\end{abstract}

JEL Codes: E22, E24, J2, C15.

Key Words: Capital Adjustment Costs, Investment, Labor Market Dynamics, Simulated Method of Moments. 


\section{Introducción}

El grado en el que las reformas políticas o el cambio en las condiciones de mercado afectan la reasignación y la demanda de factores posee implicancias en torno a la distribución de recursos. Los aspectos distributivos dependen de como ajustan los factores en respuesta al estímulo recibido, y de los costos que enfrentan los mismos para hacerlo. Entender las consecuencias generadas por reformas políticas (cambios impositivos, variaciones en la política comercial, etc.) y otros shocks que impactan sobre la economía implica reconocer la existencia de conflictos distributivos, caracterizar a los ganadores y perdedores asociados a los procesos de reasignación de recursos e identificar la dinámica de la economía en el corto y largo plazo.

En el presente trabajo se analizará el rol del capital. En particular, nos interesará entender la naturaleza de los costos de ajuste del capital y las implicancias que ellos imponen sobre la respuesta del sistema económico ante shocks, considerando tanto efectos sobre la demanda derivada de factores como así también consecuencias distributivas.

Tanto el capital como el trabajo enfrentan costos de ajuste. Si bien estimar los costos de ajuste del trabajo es un objetivo que se encuentra fuera del alcance del presente estudio, nuestros resultados destacan la importancia de los supuestos acerca del funcionamiento de los mercados laborales para conocer los efectos de los shocks sobre las variables de interés. En este sentido Peluffo (2010) presenta estimaciones de costos de ajuste del empleo, en base a un modelo estructural, utilizando datos para Argentina, encontrando altos costos de movilidad y ajustes lentos de las variables ante shocks comerciales y tecnológicos. Por otra parte, Bet, Brambilla y Porto (2011) utilizando también datos para Argentina, incorporan tanto costos de movilidad para el trabajo como costos de ajuste en el capital. Este último trabajo, destaca la importancia de modelar conjuntamente fricciones en el mercado laboral y en el mercado de capital para entender los efectos de shocks comerciales en países en desarrollo.

Sobre la base de un modelo estructural de demanda por inversión para la firma, presentaremos y discutiremos un marco que permita lograr un claro entendimiento de los efectos de shocks sobre el ajuste dinámico de la economía. En nuestro modelo, las firmas eligen período a período su nivel óptimo de inversión con el objetivo de maximizar el valor presente esperado de los beneficios intertemporales. Adicionalmente, incorporaremos como componentes sobresalientes del modelo una función de costos por ajustar el stock de capital y heterogeneidad entre firmas.

El primer objetivo del trabajo consiste en estimar los costos de ajuste del capital. Dichos costos reflejan una gran variedad de factores interdependientes que generan 
fricciones en el proceso de asignación de capital a nivel de la firma. La reasignación de factores y/o recursos por la instalación o reemplazo de capital puede ocasionar pérdidas en término del producto. Asimismo, la instalación de una nueva estructura de producción usualmente conlleva costos de reentrenamiento, de instalación y de prueba. La ausencia o imperfecciones de mercados secundarios para ciertos bienes de capital también genera costos de ajuste dada la irreversibilidad (parcial o total) que implica la instalación de dicho capital en la planta.

Tradicionalmente la literatura ha analizado la estructura y magnitud de los costos de ajuste asumiendo una forma funcional convexa (usualmente cuadrática) para los mismos. Sin embargo, en los últimos años, la literatura ha resaltado el rol de las noconvexidades y de las irreversibilidades a nivel de la firma (o planta) en el proceso de inversión. ${ }^{1}$ El énfasis puesto en estas estructuras de costos radica en que la evidencia a nivel de la planta de producción indica la existencia de una relación no lineal entre la tasa de inversión y ciertos momentos relevantes de la misma. Más precisamente, ante la ausencia de costos de ajuste, la inversión respondería excesivamente a los shocks y presentaría una correlación serial negativa en caso que los shocks exhiban cierto grado de persistencia (algo que no se observa en los datos a nivel de la planta). En este sentido, el rol de los costos de ajuste consiste en morigerar la respuesta de la inversión y crear una correlación serial positiva en dicha variable. Por otra parte, si bien los costos de ajuste convexos son capaces de reproducir correlación serial positiva en las tasas de inversión, no logran reproducir otras características observadas en los datos tales como episodios de gran inversión, desinversión, o inacción (tasas de inversión nulas). La incorporación de componentes no-convexos e irreversibilidades en la forma funcional de los costos de ajuste busca remediar estas falencias logrando una mejor aproximación a las características de la tasa de inversión observadas en los datos.

Se estimarán los parámetros correspondientes a los costos de ajuste del capital haciendo uso de un modelo estructural dinámico y considerando los distintos componentes discutidos por la literatura para la función de costos (convexidades, no-convexidades, e irreversibilidades). La estrategia de estimación se basará en comparar el comportamiento de la tasa de inversión predicho por el modelo, con la dinámica observada para la inversión en los datos para un panel de plantas manufactureras argentinas para el período 1994-2001. Para ello haremos uso del método de momentos simulados (MMS) el cual minimiza la distancia ponderada entre momentos observados en los datos y momentos

\footnotetext{
${ }^{1}$ Véase por ejemplo Abel y Eberly (1994), Caballero, Engel y Haltiwanger (1995), Abel y Eberly (1996), Doms y Dunne (1998), Cooper, Haltiwanger y Power (1999), Huggett y Ospina (2001), Nilsen y Schiantarelli (2003), y Cooper y Haltiwanger (2006)
} 
simulados por el modelo.

El segundo objetivo del presente estudio consiste en realizar un análisis ex-ante de los efectos potenciales de shocks sobre los mercados de factores. Para ello, utilizaremos las estimaciones de los parámetros del modelo con el objetivo de realizar simulaciones contrafácticas de los efectos sobre la asignación de capital, trabajo y salarios de una reducción en la rentabilidad media de las firmas.

Los resultados obtenidos destacan la relevancia de las convexidades, no-convexidades e irreversibilidades en la estructura de costos de ajuste a nivel de las plantas. Los parámetros estimados implican grandes costos fijos e irreversibilidades, junto con moderados costos cuadráticos.

Asimismo, los ejercicios de simulación resaltan la importancia de modelar conjuntamente las decisiones de inversión de las firmas y las fricciones en la movilidad del capital a fines de analizar los efectos de shocks sobre los mercados de factores. En particular, los resultados sugieren que ante costos de ajuste del capital el ajuste de la economía en respuesta a un shock es lento (contrastando a los resultados obtenidos luego de asumir inexistencia de costos de ajuste o inmovilidad en el capital). Adicionalmente, los efectos del shock sobre el salario, el empleo, y el capital agregado son de magnitudes considerables, superando las obtenidas asumiendo inmovilidad del capital.

El trabajo se organiza de la siguiente manera. En la sección 2 se desarrolla un modelo teórico de demanda de capital sobre la base del cual serán formulados los resultados. La sección 3 describe los datos utilizados en el análisis empírico, mientras que la sección 4 presenta la estrategia de estimación junto con los resultados obtenidos. En la sección 5 se discuten los resultados de los ejercicios de simulación realizados. Finalmente, las principales conclusiones se presentan en la sección 6 .

\section{El Modelo}

En esta sección se presenta el modelo estructural de demanda por inversión intertemporal que será utilizado para estimar los parámetros de costos de ajuste del capital a nivel de la firma.

Las firmas eligen el nivel óptimo de factores variables (trabajo y materiales) con el objetivo de maximizar beneficios instantáneos período a período. Asimismo, en cada período, las firmas escogen su nivel de inversión óptimo a fines de maximizar los beneficios intertemporales descontados. Mientras que los factores variables pueden ser 
ajustados libremente, existen costos por variar el stock de capital. La solución al problema de optimización dinámico otorga como resultado una función de demanda por inversión que depende de las variables de estado del problema. A continuación se discuten en detalle los distintos componentes del modelo.

\subsection{Producción y Demanda}

Las firmas producen utilizando una tecnología de producción Cobb-Douglas de la forma:

$$
Q_{i t}=\widetilde{A}_{i t} K_{i t}^{\alpha_{k}} L_{i t}^{\alpha_{l}} M_{i t}^{\alpha_{m}}
$$

donde $\widetilde{A}_{i t}$ representa un shock a la productividad (idiosincrásico) enfrentado por la firma $i$ en el período $t, K_{i t}$ el nivel de capital, $L_{i t}$ el nivel de empleo demandado, y $M_{i t}$ el nivel de materiales o insumos intermedios empleados. $\alpha_{l}, \alpha_{m}$ y $\alpha_{k}$ son parámetros estimables.

El tiempo es discreto y el horizonte temporal de la firma es infinito. Adicionalmente, la inversión se torna productiva con un período de retraso, mientras que el capital se deprecia al final de cada período. De esta forma, la ley que gobierna la acumulación de capital de la firma puede escribirse como:

$$
K_{i, t+1}=(1-\delta) K_{i t}+I_{i t}
$$

donde $I_{i t}$ representa la inversión realizada por la firma $i$ en el período $t$, mientras que $\delta$ es la tasa de depreciación del capital.

La demanda de mercado es isoelástica. Esto implica que las firmas enfrentarán curvas de ingreso marginal con pendiente negativa. Dicha demanda de mercado está representada por:

$$
Q_{i t}=D_{i t} P_{i t}^{-\varepsilon}
$$

donde $\varepsilon>1$ es la elasticidad de demanda, $D_{i t}$ es un parámetro que mide el tamaño de mercado en el cual la firma participa, y $P_{i t}$ representa el precio fijado por la firma. ${ }^{2}$

Dado que las firmas no enfrentan costos por ajustar su nivel de empleo y materiales,

\footnotetext{
2 Notar que el supuesto de demandas isoelásticas implica que las firmas sean heterogéneas produciendo productos diferenciados.
} 
la función de beneficios corrientes puede ser expresada como: ${ }^{3}$

$$
\pi_{i t}\left(A_{i t}, K_{i t}\right)=A_{i t} K_{i t}^{\theta}
$$

donde $A_{i t}$ es un shock de rentabilidad enfrentado por la firma $i$ en el período $t$, compuesto por variaciones en el costo del empleo $\left(w_{t}\right)$, variaciones en el costo unitario de los materiales $\left(z_{t}\right)$, variaciones en la productividad factorial $\left(\widetilde{A_{i t}}\right)$, y variaciones en la demanda de mercado $\left(D_{i t}\right)$. Asimismo, $\theta$ es un parámetro compuesto por parámetros tecnológicos y la elasticidad de demanda de mercado, siendo de esta forma estimable. ${ }^{4}$

\subsection{Shocks de Rentabilidad}

Como fue mencionado previamente, las variaciones en el costo del empleo $\left(w_{t}\right)$, en el costo unitario de los materiales $\left(z_{t}\right)$, en la productividad factorial $\left(\widetilde{A_{i t}}\right)$, y en la demanda de mercado $\left(D_{i t}\right)$ son las cuatro fuentes de incertidumbre en el modelo. Se representará a estas cuatro fuentes de incertidumbre mediante una sola variable $\left(A_{i t}\right)$ que capturará los diferentes estados de rentabilidad de la firma. Siguiendo la literatura de costos de ajuste, se asume que el logaritmo natural de $A_{i t}$ evoluciona de acuerdo a un proceso de Markov. Llamaremos $\mathcal{Q}$ a la función de transición asociada a $a_{i t}=\ln A_{i t}$.

Los supuestos realizados sobre la evolución de $a_{i t}$ implican que los shocks de rentabilidad poseen efectos que son persistentes pero no permanentes. Adicionalmente, a fines de decidir el nivel óptimo de inversión, las firmas toman en consideración su estado de rentabilidad vigente en el período $t\left(A_{i t}\right)$, pero poseen incertidumbre acerca de los estados de rentabilidad futuros, los cuales dependen de las realizaciones futuras de los shocks de productividad y demanda, así como del nivel de salarios y costo unitario de los materiales.

\subsection{Costos de Ajuste del Capital}

La demanda por inversión de la firma depende tanto de los estados de rentabilidad enfrentados por la firma como de los costos de ajustar el capital. La literatura reconoce tres tipos de costos en el ajuste del capital: costos fijos, costos cuadráticos e irreversibilidades parciales.

\footnotetext{
${ }^{3}$ La función de beneficios corrientes de la firma surge de optimizar los beneficios instantáneos escogiendo el nivel de insumos variables, dado el stock de capital y estado de rentabilidad corriente. El apéndice A.1 detalla la derivación de los resultados presentados en esta sección.

${ }^{4} \mathrm{El}$ apéndice A.1 detalla la formas funcionales correspondientes a $A_{i t} \mathrm{y} \theta$.
} 


\subsubsection{Costos Fijos}

Los costos fijos son independientes del nivel de inversión, y son pagados en aquellos períodos de tiempo en los cuales la firma invierte (o desinvierte). Este componente se vincula a las no convexidades en la forma funcional de los costos de ajuste. Cooper y Haltiwanger (2006) señalan que dichos costos capturan indivisibilidades en el capital, retornos crecientes en la instalación de nuevo capital, y retornos crecientes en la reestructuración de la actividad productiva.

Modelaremos a los costos fijos como proporcionales al stock de capital corriente de la firma. Es interesante destacar que estos costos se vuelven relevantes cuando la firma incrementa su stock de capital. Asimismo, los costos fijos pueden ser evitados escogiendo un nivel de inversión nulo. ${ }^{5}$

La forma funcional asumida para los costos fijos está dada por:

$$
C_{i t}^{f}\left(K_{i t}, I_{i t}, A_{i t}\right)=f 1_{\left[I_{i t} \neq 0\right]} K_{i t}
$$

donde $1_{\left[I_{i t} \neq 0\right]}$ es una variable indicadora igual a uno si la inversión es distinta de cero, mientras que $f$ representa un parámetro que refleja la magnitud de los costos fijos en términos del capital promedio de la firma.

\subsubsection{Costos Cuadráticos}

Los costos de ajuste cuadráticos son aquellos que aumentan (a tasa creciente) con el nivel de la tasa de inversión. La especificación asumida contempla las siguientes características: (i) en primer lugar, una especificación cuadrática en la tasa de inversión, asociando costos más altos con cambios abruptos en el nivel de inversión (es decir, una tasa de inversión o desinversión elevada); (ii) en segundo lugar, la función alcanza su valor mínimo para un nivel de inversión nulo; (iii) finalmente, los costos son

\footnotetext{
${ }^{5}$ Los mismos pueden ser modelados como proporcionales al nivel de ingreso de la firma. Véase por ejemplo Bloom (2009), Cooper y Haltiwanger (2006) o Caballero y Engel (1999). Estos últimos autores estiman un modelo en el cual los costos fijos son aleatorios. La modelización de los costos fijos como proporcionales al ingreso implica una caída en la productividad de la firma durante períodos de inversión. De esta forma, grandes inversiones serán menos costosas durante períodos de baja rentabilidad, dado que los costos de ajuste también serán más bajos. Esto no necesariamente implica una correlación negativa entre la inversión y los shocks de rentabilidad, ya que existirá una ganancia por invertir en estados de alta rentabilidad si la correlación serial de los shocks de rentabilidad es lo suficientemente alta. Optamos por no incorporar dicha especificación en el modelo porque la misma implica cierto grado de distorsión en la medida de los shocks de rentabilidad. En particular, en caso de modelar los costos fijos como proporcionales al nivel de ingreso de la firma, sería necesario emplear una estrategia de estimación que permita distinguir los costos de ajuste de realizaciones bajas de los shocks sobre la función de beneficios.
} 
proporcionales al stock de capital de la firma.

La forma funcional asumida para los costos de ajuste cuadráticos está dada por:

$$
C_{i t}^{q}\left(K_{i t}, I_{i t}, A_{i t}\right)=\frac{\gamma}{2}\left(I_{i t} / K_{i t}\right)^{2} K_{i t}
$$

donde $\gamma$ es un parámetro que mide la magnitud de los costos convexos.

\subsubsection{Irreversibilidades Parciales}

El tercer componente asociado a los costos de ajuste se vincula con la existencia de costos de transacción: costos asociados a la reventa del bien, a la especificidad del capital, y a las asimetrías de información en la venta de bienes de capital. Dichos costos son modelados mediante una brecha entre el precio de compra del capital $\left(p_{b}\right)$ y el precio de venta $\left(p_{s}\right)$. En particular, incorporamos los mismos dentro del modelo asumiendo $p(I)=p_{b}$ si $I_{i t}>0$ y $p(I)=p_{s}$ si $I_{i t}<0$, donde $p_{b} \geq p_{s}$.

Esta clase de costos de ajuste crea una región de inacción para la firma dado que la misma responderá ante un shock adverso manteniendo capital (para evitar las pérdidas asociadas a la reventa del mismo), mientras que por otro lado, no incrementará rápidamente su stock de capital en períodos de alta rentabilidad dado el costo de reventa del mismo.

La forma funcional asumida para la irreversibilidad parcial esta dada por:

$$
C_{i t}^{I}\left(K_{i t}, I_{i t}, A_{i t}\right)=\left\{\begin{array}{l}
p_{b} I_{i t} 1_{\left[I_{i t}>0\right]} \\
p_{s} I_{i t} 1_{\left[I_{i t}<0\right]}
\end{array}\right.
$$

donde $1_{\left[I_{i t}>0\right]}$ y $1_{\left[I_{i t}<0\right]}$ son variables indicadoras iguales a uno si la inversión es estrictamente positiva y negativa respectivamente.

El modelo incorpora las tres formas funcionales comentadas. De esta forma, la función de costos de ajuste puede ser representada mediante la siguiente forma funcional:

$$
C_{i t}\left(K_{i t}, I_{i t}, A_{i t}\right)=\left\{\begin{array}{lll}
\frac{\gamma}{2}\left(I_{i t} / K_{i t}\right)^{2} K_{i t}+f K_{i t}+p_{b} I_{i t} & \text { si } & I_{i t}>0 \\
0 & \text { si } & I_{i t}=0 \\
\frac{\gamma}{2}\left(I_{i t} / K_{i t}\right)^{2} K_{i t}+f K_{i t}+p_{s} I_{i t} & \text { si } & I_{i t}<0
\end{array}\right.
$$

donde el vector de parámetros $\left(f, \gamma, p_{b}, p_{s}\right)$ caracteriza la estructura de costos de ajuste de la firma. 


\subsection{Inversión óptima}

Luego de haber definido los componentes que forman parte del problema enfrentado por la firma, es posible caracterizar la solución del mismo. En particular, el problema consiste en maximizar el valor presente esperado de los beneficios de la firma neto de los costos de ajuste, eligiendo el nivel de inversión $\left(I_{i t}\right)$, la cual será productiva con un período de retraso. Formalmente:

$$
\begin{array}{r}
\operatorname{máx}_{I_{i t}} E_{t} \sum_{t=0}^{\infty} \beta^{t}\left(\pi_{i t}\left(K_{i t}, A_{i t}\right)-C_{i t}\left(K_{i t}, I_{i t}, A_{i t}\right)\right) \\
\text { s.a. : } K_{i, t+1}=(1-\delta) K_{i t}+I_{i t}
\end{array}
$$

donde $\beta \in(0,1)$ es un factor de descuento, $\delta$ es la tasa de depreciación del capital, y $E_{t}$ representa el operador esperanza condicional a la información disponible en $t$.

A fines de caracterizar la inversión óptima, notemos que el problema representado por las ecuaciones (2-8) y (2-9) es equivalente a la función de valor que resuelve la ecuación de Bellman:

$$
V_{i t}\left(K_{i t}, A_{i t}\right)=\operatorname{máx}_{I_{i t}} u_{i t}\left(K_{i t}, I_{i t}, A_{i t}\right)+\beta E_{t} V_{i, t+1}\left(K_{i, t+1}, A_{i, t+1}\right)
$$

donde,

$$
\begin{aligned}
u_{i t}\left(K_{i t}, I_{i t}, A_{i t}\right) & =\pi_{i t}\left(K_{i t}, A_{i t}\right)-C_{i t}\left(K_{i t}, I_{i t}, A_{i t}\right) \\
E_{t} V_{i, t+1}\left(K_{i, t+1}, A_{i, t+1}\right) & =\int V_{i, t+1}\left(K_{i, t+1}, A_{i, t+1}\right) \mathcal{Q}\left(A, d A_{t+1}\right)
\end{aligned}
$$

siendo $\mathcal{Q}($.$) la función de transición del proceso de Markov A_{i t}$.

La solución a la ecuación de Bellman (2-10) es provista por la siguiente regla de decisión:

$$
I_{i t}=g\left(K_{i t}, A_{i t}\right)
$$

la cual es una función de las variables de estado $(K, A)$ de la firma. Esta función, le indica a la firma cuál debe ser su nivel de inversión óptimo dado su stock de capital $(K)$ y estado de rentabilidad $(A)$ corriente. 


\section{Datos}

La presente sección describe los datos utilizados en el análisis empírico. La base de datos utilizada consiste en un panel balanceado para un total de 568 plantas manufactureras Argentinas, abarcando el período 1994-2001. Los mismos fueron relevados por la Encuesta Industrial Anual (EIA). Los datos contienen información relativa a variables vinculadas al valor bruto de producción, valor agregado, costos, empleo, consumo de energía y combustibles, stock de existencias, y compra y venta de bienes de capital. Asimismo, la base de datos no registra información del stock de capital a nivel de planta, siendo necesario generar esta variable. El apéndice A.3 describe el procedimiento utilizado para construir la serie de stock de capital real a nivel de la planta. ${ }^{6}$

La tabla 1 presenta estadísticas descriptivas para algunas variables. La misma provee información relativa al valor bruto de producción, valor agregado, stock de capital, inversión y materiales (todas ellas expresadas en miles de pesos constantes de 1993). La tabla brinda también información respecto al número de empleados afectados al proceso productivo.

Una gran ventaja a la hora de analizar la naturaleza de los costos de ajuste a nivel de la planta reside en que los datos contienen información tanto para compras como para ventas de bienes de capital. En particular, esto hace posible analizar tanto ajustes positivos como negativos en el stock de capital, permitiendo el análisis relativo al rol de las irreversibilidades parciales y los costos de transacción (que sería inviable en caso contrario).

Dado el interés del trabajo en la estimación de los costos que afectan la acumulación de capital, nos concentraremos de aquí en adelante en la tasa de inversión, definida como el cociente entre la inversión real neta y el stock real de capital $\left(I_{i t} / K_{i t}\right)$. El gráfico 1 presenta la distribución de la tasa de inversión observada para el período 1994-2001 y el total de plantas manufactureras. A dicha distribución se le superpone un gráfico de la distribución normal. Se observa que la tasa de inversión presenta una gran densidad alrededor del cero, largas colas y una fuerte asimetría hacia la derecha, difiriendo notablemente de la distribución normal.

La tabla 2 presenta información respecto a ciertos momentos relevantes de la tasa de inversión, que complementa la información provista por el gráfico 1. Esta presenta

\footnotetext{
${ }^{6} \mathrm{El}$ panel balanceado evita que sea necesario modelar la entrada y salida al mercado de las firmas. Sin embargo, este implica que las estimaciones obtenidas contengan potencialmente un sesgo por selección. La decisión de utilizar un panel balanceado de plantas radica en la dificultad de medir el stock real de capital y generar la serie para esta variable en el caso de utilizar un panel no balanceado. Véase el apéndice A.3 para una descripción del procedimiento utilizado para construir la serie de stock de capital real.
} 
información respecto a ciertos momentos relevantes de la tasa de inversión. Observamos que, para el período 1994-2001, la tasa de inversión media es igual a $9.71 \%$ con un desvío estándar de $22.89 \%$, implicando una gran dispersión en la variable. Tanto el signo como la magnitud del coeficiente de asimetría (6.862) indican una gran asimetría en la distribución, con una larga cola hacia la derecha. Asimismo, observamos un gran porcentaje de inacción (esto es, tasas de inversión menores al $1 \%$ en valor absoluto), totalizando un $29.17 \%$ para la muestra completa. Por el contrario, observamos bajas tasas de inversión negativa (tasas de inversión menores al -1\%) al igual que episodios de ajustes abruptos en el stock de capital a nivel de la planta mediante la venta de bienes de capital (en promedio, sólo el $2.71 \%$ del total de observaciones (plantas-año) exhiben tasas de inversión menores al $-5 \%$ ).

Los datos sugieren que la industria se caracteriza por ajustes abruptos y positivos en el stock de capital. El $13.57 \%$ de las observaciones contenidas en la muestra presentan tasas de inversión de al menos un 20\%. En promedio, estos episodios de gran inversión representan aproximadamente el $57.18 \%$ del monto total de inversión para toda la muestra. Este hallazgo, junto con los comentados en el párrafo anterior, ilustran la gran asimetría existente en los datos.

La tabla 2 también reporta la correlación serial de la tasa de inversión, computada como la correlación simple entre la tasa de inversión a nivel de la planta y su rezago. Si bien la correlación serial es positiva, esta no es elevada (0.1870). La segunda medida de correlación presentada en la tabla se corresponde con la correlación entre la tasa de inversión y los shocks de rentabilidad. Estimamos dicho momento en 0.1803, indicando que las tasas de inversión son mayores en períodos de alta rentabilidad. ${ }^{7}$ Cabe resaltar, que estas dos medidas de correlación han sido enfatizadas en la literatura de costos de ajustes como sensibles a la estructura de costos de ajuste del capital a nivel de la planta.

Finalmente, la tabla 2 exhibe el valor de los momentos para algunos años en particular. Se observa para cada uno de ellos un comportamiento similar al observado para el total del período (1994-2001). La excepción la constituye el año 2001, en donde se registran algunos cambios significativos. En particular, tanto la tasa de inversión media como los episodios de gran inversión caen a un $4.23 \%$ y $6.57 \%$ respectivamente. Asimismo, el coeficiente de asimetría cae notablemente (volviéndose negativo aunque cercano a cero) mientras que la inacción y la fracción de observaciones con tasa de inversión negativa aumentan respecto a los valores obtenidos para el total del período.

\footnotetext{
7 A fines de computar este último momento, es necesario contar con una medida para los shocks de rentabilidad. La sección 4.3 describe la construcción de dicha medida.
} 
Estos cambios están asociados a la profunda crisis económica atravesada por el país en el año 2001.

\section{Estimación}

Esta sección describe la metodología utilizada para estimar los parámetros del modelo. El conjunto total de parámetros a estimar está representado por el vector $\Theta$ que caracteriza la función de beneficios de la firma, la función de costos de ajuste y el proceso estocástico, junto con la tasa de descuento y depreciación del capital. El problema econométrico consiste en estimar dicho vector de parámetros. Dado que el modelo no posee una solución en forma cerrada, los parámetros no pueden ser estimados con técnicas econométricas estándar.

La literatura reciente ha estimado este tipo de modelos dinámicos estocásticos, que incorporan funciones de costos tanto convexas como no convexas, utilizando el método de momentos simulados (véase por ejemplo Cooper y Haltiwanger (2006), Bloom (2009) o Bond, Soderbom y Wu (2008) para estimaciones de costos de ajuste del capital; o Cooper, Haltiwanger y Willis (2004), Ejarque y Portugal (2007) o Ejarque y Nilsen (2008) para estimaciones de costos de ajuste del trabajo). En principio, la totalidad de los parámetros del modelo podrían ser estimados simultáneamente utilizando el método de momentos simulados. Sin embargo, esta estrategia impone un gran número de restricciones sobre los datos a ser utilizados e implica en la práctica un gran esfuerzo computacional.

A fines de simplificar la estimación, utilizamos una estrategia similar a la utilizada por Cooper y Haltiwanger (2006). En particular, hacemos uso de diferentes metodologías con el objetivo de recuperar los parámetros asociados a la función de producción, a los shocks de rentabilidad, y a la elasticidad de demanda. A su vez, fijamos la tasa de descuento $(\beta)$ en 0.95 y la tasa de depreciación del capital $(\delta)$ igual al valor de la media (ponderada) de las tasas de depreciación utilizadas para la construcción de la serie de stock de capital. ${ }^{8}$ Por último, los parámetros de costos de ajuste del capital son estimados a través del método de momentos simulados. Para ello, establecemos $p_{b}=1$ con el objetivo de interpretar las estimaciones sobre el parámetro $p_{s}$ como pérdidas porcentuales por la reventa de bienes de capital.

\footnotetext{
${ }^{8}$ Como es mencionado en el apéndice A.3, a fines de construir la serie de stock de capital, utilizamos información de tasas de depreciaciones con un nivel de desagregación a 2 dígitos para la industria manufacturera.
} 
A continuación se discute en detalle la metodología de estimación y los resultados correspondientes a cada conjunto de parámetros.

\subsection{Producción}

El estado de rentabilidad de la planta es una variable de estado en el problema de decisión de la firma que no es observada por el econometrista. Esto genera inconvenientes en la estimación de los parámetros asociados a la función de producción de la planta. En particular, las estimaciones mínimo cuadráticas contienen un sesgo de simultaneidad, dado que los factores variables se encuentran correlacionados con la productividad inobservada. Si plantas con mayor productividad o rentabilidad demandan mayores insumos, entonces las estimaciones mínimo cuadráticas de los coeficientes estarían sesgadas hacia arriba. ${ }^{9}$ Por este motivo estimaremos los coeficientes asociados a la función de producción mediante el estimador desarrollado por Olley y Pakes (1996). Este estimador, utilizado recurrentemente en la literatura, permite controlar el problema de simultaneidad utilizando la inversión como proxy de los shocks de productividad inobservables y variantes en el tiempo. El apéndice A.4 provee detalles adicionales vinculados al estimador y a la implementación del mismo.

La tabla 3 presenta los resultados obtenidos para los coeficientes asociados a la función de producción especificada en la ecuación (2-1). Realizamos las estimaciones utilizando los estimadores de mínimos cuadrados ordinarios, efectos fijos y Olley-Pakes. De acuerdo a la teoría, los coeficientes asociados a los factores variables (en este caso trabajo y materiales) deberían contener un sesgo hacia arriba en las estimaciones mínimo cuadráticas. Este efecto debería ser mayor en factores que son más fáciles de ajustar. Las estimaciones obtenidas bajo el estimador desarrollado por Olley-Pakes para los coeficientes de las variables trabajo, materiales y capital difieren de las obtenidas bajo los estimadores de efectos fijos y de mínimo cuadrados ordinarios. La dirección del movimiento apunta en el sentido de la eliminación de los sesgos de simultaneidad (a excepción del factor trabajo cuyo coeficiente estimado bajo Olley-Pakes resulta superior al obtenido bajo mínimos cuadrados ordinarios). Los coeficientes estimados a través de

\footnotetext{
9 Asimismo, existe también un problema de selección, dado que el econometrista sólo observa aquellas plantas con la productividad suficiente como para permanecer en el mercado. Si los estados de rentabilidad de la plantas se relacionan positivamente con el stock de capital de estas, entonces plantas con mayor capital poseen una probabilidad mayor de permanecer en el mercado (dada la posibilidad de poder enfrentar un estado de rentabilidad bajo en el futuro). Esto implica un sesgo hacia abajo en los coeficientes asociados al capital en las estimaciones mínimo cuadráticas. El hecho de que nuestras estimaciones se realicen sobre la base de un panel balanceado de plantas, implica que las mismas contengan potencialmente un sesgo por selección.
} 
Olley-Pakes para los factores trabajo y materiales son iguales a 0.217 y 0.676 , respectivamente. Dichos coeficientes son estimados en 0.202 y 0.709 en el caso de mínimos cuadrados ordinarios, y en 0.290 y 0.552 para el estimador de efectos fijos. En el caso del coeficiente asociado al capital, el resultado estimado mediante Olley-Pakes (0.091) resulta inferior al obtenido mediantes mínimo cuadrados ordinarios (0.093) y superior al obtenido bajo efectos fijos (0.039).

Los coeficientes obtenidos bajo el estimador de efectos fijos resultan considerablemente más bajos comparados con los obtenidos bajo mínimos cuadrados ordinarios u Olley-Pakes (exceptuando los obtenidos para el factor trabajo). Estas diferencias son más notables en el caso del capital. Este resultado no es sorprendente dada la naturaleza del estimador, la cual subyace en la variación intertemporal dentro de la planta, incrementando de esta forma la importancia de los errores de medición. Por último, cabe resaltar que los resultados obtenidos son comparables a los obtenidos por otros autores para el caso de países en desarrollo (véase por ejemplo Pavcnik (2000) para el caso de Chile).

\subsection{Demanda}

Estimamos la elasticidad de demanda enfrentada por las firmas explotando la información sobre ingresos y costos contenida en nuestra base de datos junto con la relación entre ingresos, costos y elasticidad de demanda implicada por la maximización de beneficios corrientes y derivada en el apéndice A.2:

$$
\frac{R_{i t}-c_{i t} / \nu}{R_{i t}}=\varepsilon_{i t}^{-1}
$$

donde $R_{i t}$ representa los ingresos por ventas de la firma $i$ en el período $t, c_{i t}$ los costos variables (asociados al factor trabajo e insumos intermedios), mientras que $\nu=\left(\alpha_{l}+\right.$ $\left.\alpha_{m}\right)$.

La ecuación (4-1) provee las bases para la estimación de la elasticidad de demanda. En particular, estimamos dicha elasticidad como el promedio de las elasticidades medias específicas de cada una de las plantas:

$$
(\varepsilon)^{-1}=\frac{1}{N} \sum_{i=1}^{N}\left(\frac{1}{T} \sum_{t=1}^{T} \frac{R_{i t}-c_{i t} / \nu}{R_{i t}}\right)
$$

donde $N$ representa el número total de plantas, y $T$ el número total de períodos.

La tabla 4 reporta el valor estimado para la elasticidad de demanda de mercado 
$(\varepsilon)$. Estimamos el valor de dicho parámetro en 5.284. Esto implica un markup para el total de la industria manufacturera de aproximadamente un $19 \%{ }^{10}$

\subsection{Shocks de Rentabilidad}

La estructura de los shocks de rentabilidad $A_{i t}$ constituye una pieza fundamental en la estimación de los parámetros de costos de ajuste del capital. Siguiendo a Cooper y Haltiwanger (2006), los shocks de rentabilidad son modelados mediante la interacción de un shock agregado $\left(b_{t}\right)$ y un shock idiosincrásico $\left(e_{i t}\right)$ :

$$
\ln A_{i t}=b_{t}+e_{i t}
$$

Obtenemos una medida para $A_{i t}$ haciendo uso de la ecuación (2-3), junto con la información contenida en los datos referida a los beneficios operativos de la firma $\left(\pi_{i t}\right)$, el stock de capital $\left(K_{i t}\right)$, y las estimaciones de la función de producción y elasticidad de demanda (contenidas en $\theta$ ). Computamos $b_{t}$ como la media (por año) de $\ln A_{i t}$, mientras que el componente idiosincrásico $\left(e_{i t}\right)$ está dado por la diferencia entre estas dos medidas.

Modelamos $b_{t}$ como un proceso de Markov de primer orden, con dos estados, y matriz de transición simétrica. Tanto los estados como la matriz de transición son elegidos de modo tal de reproducir el grado de correlación serial y el desvío estándar de las innovaciones observados en los datos. De esta forma, establecemos una matriz de transición cuyos elementos diagonales igualan 0.8 , mientras que los estados del shock son elegidos de modo tal de reproducir el desvío estándar del proceso observado en los datos.

Se asume que $e_{i t}$ sigue un proceso de Markov autorregresivo de primer orden $(\mathrm{AR}(1))$ dado por:

$$
e_{i t}=\mu_{e}+\rho_{e} e_{i, t-1}+\zeta_{i t}
$$

donde $\zeta_{i t} \sim N\left(0 ; \sigma_{e}\right), \mathrm{y} \rho_{e}$ representa el coeficiente de autocorrelación de primer orden del proceso. Los momentos $\sigma_{e} \mathrm{y} \rho_{e}$ son críticos a la hora de entender ciertos momentos

\footnotetext{
${ }^{10}$ Los resultados son comparables con los obtenidos por otros trabajos. Bond et al. (2008) estiman una elasticidad de demanda igual a 6.38 utilizando un panel de firmas manufactureras del Reino Unido para el período 1972-1991. Cooper y Haltiwanger (2006), utilizando datos de plantas manufactureras estadounidenses para el período 1972-1988, infieren una elasticidad de demanda igual a 6.2. Finalmente, Bloom (2009) asume un valor de elasticidad de demanda de 4 para sus estimaciones de costos de ajuste del capital y empleo en base a un panel de firmas estadounidenses para el paríodo 1981-2000.
} 
asociados a la tasa de inversión, tales como los episodios de gran inversión o inacción. En particular, estos parámetros determinan el riesgo enfrentado por la firma: un mayor grado de autocorrelación y una menor varianza de las innovaciones permiten a la firma realizar predicciones más precisas de los shocks de rentabilidad futuros. De esta forma, dichos parámetros determinan las decisiones óptimas de la firma para una estructura de costos de ajuste determinada, como así también los beneficios de reasignación de factores entre firmas. Adicionalmente, a nivel macro, estos parámetros afectan la productividad factorial total y pueden afectar la dinámica del ciclo económico (Gourio (2008)).

Luego de computar el componente idiosincrásico de los shocks de rentabilidad, estimamos la ecuación (4-4) por mínimos cuadrados ordinarios, con el objetivo de recuperar los parámetros de interés. La tabla 4 presenta los momentos estimados asociados al componente idiosincrásico de los shocks de rentabilidad. Los shocks idiosincrásicos a nivel de la planta están altamente correlacionados. El parámetro $\rho_{e}$ es estimado en 0.883 para toda la industria. Adicionalmente, la varianza de las innovaciones asociadas al componente idiosincrásico de los shocks también resulta ser alta. El desvío estándar de las innovaciones de dicho componente $\left(\sigma_{e}\right)$ es estimado en 0.699.

\subsection{Costos de Ajuste del Capital}

Estimamos los parámetros de costos de ajuste del capital utilizando el método de momentos simulados. Este método minimiza una función de distancia compuesta de momentos observados en los datos y momentos obtenidos a partir de datos simulados. ${ }^{11}$ Para un valor dado del vector de parámetros $\Theta_{K}=\left(f, \gamma, p_{s}\right)$ el problema dinámico es resuelto y las reglas de decisión óptima para la firma son obtenidas. Las reglas de decisión óptima son utilizadas para generar una versión simulada de los datos. Los primeros 100 períodos son eliminados para evitar que los resultados dependan de las condiciones iniciales asumidas. Luego, un conjunto de momentos $\Psi^{s}(\theta)$ son calculados de los datos simulados.

La función de pérdida $\Gamma\left(\Theta_{K}\right)$ asociada a dichos momentos simulados es construida del siguiente modo:

$$
\Gamma\left(\Theta_{K}\right)=\left[\Psi^{d}-\Psi^{s}\left(\Theta_{K}\right)\right]^{\prime} W\left[\Psi^{d}-\Psi^{s}\left(\Theta_{K}\right)\right]
$$

El parámetro $\widehat{\Theta_{K}}$ es derivado entonces como el que minimiza la distancia ponderada

\footnotetext{
11 Para una discusión en detalle del método de momentos simulados y sus propiedades estadísticas véase McFadden (1989) y Pakes y Pollard (1989).
} 
entre los momentos observados en los datos $\left(\Psi^{d}\right)$ y los momentos simulados $\left(\Psi^{s}\left(\Theta_{K}\right)\right)$. Formalmente,

$$
\widehat{\Theta_{K}}=\arg \min _{\Theta_{K}}\left[\Psi^{d}-\Psi^{s}\left(\Theta_{K}\right)\right]^{\prime} W\left[\Psi^{d}-\Psi^{s}\left(\Theta_{K}\right)\right]
$$

donde W es una matriz de ponderación. En particular, usamos la matriz de ponderación óptima, que está dada por la inversa de la matriz de varianzas y covarianzas de los momentos observados. Lee y Ingram (1991) muestran que bajo la hipótesis nula, la matriz de varianzas y covarianzas de los momentos simulados converge a $\frac{1}{n} \Omega$, donde $\Omega$ es igual a la matriz de varianzas y covarianzas de los momentos observados y $n=N_{s} / N_{o}$, siendo $N_{s}$ la longitud de los datos simulados y $N_{o}$ la longitud de los datos observados. Asimismo, la elección eficiente de W está representada por la inversa de la matriz de varianzas y covarianzas de $\left[\Psi^{d}-\Psi^{s}\left(\Theta_{K}\right)\right]$. De esta forma, dado que $\Psi^{d}$ y $\Psi^{s}\left(\Theta_{K}\right)$ son independientes por construcción, $W=[(1+1 / n) \Omega]^{-1}$. Computamos la matriz de varianzas y covarianzas de los momentos observados $(\Omega)$ mediante bootstrap. El mismo es realizado sobre plantas, de modo tal que cuando una planta es escogida todas las observaciones correspondientes a la misma son incluídas. ${ }^{12}$

Dado que la función $\Psi^{s}\left(\Theta_{K}\right)$ no es analíticamente derivable, la minimización es realizada haciendo uso de técnicas numéricas. En particular, se utilizará un algoritmo denominado simulated annealing para la búsqueda de los parámetros, dadas las potenciales discontinuidades del modelo y la discretización del espacio de las variables de estado del problema. ${ }^{13}$

La identificación de los parámetros y la eficiencia del estimador dependen crucialmente de la elección apropiada de los momentos. Esto implica que los momentos considerados deben ser informativos acerca del cambio en los parámetros estructurales a ser estimados. La literatura sostiene que es apropiado elegir una combinación de momentos que describan tanto el comportamiento de la tasa de inversión a través del tiempo como en un período de tiempo determinado.

En base a las observaciones realizadas en la sección 3, y considerando también los argumentos discutidos en la literatura (Cooper y Haltiwanger (2006), Bloom (2009), Caballero y Engel (2003), Cooper et al. (1999)) se seleccionan cuatro momentos. Los dos primeros momentos han sido utilizados recurrentemente en trabajos empíricos que estudian los costos de ajuste, siendo ambos sensibles a la estructura de dichos costos.

\footnotetext{
${ }^{12}$ Fueron realizadas 1000 replicaciones a fines de construir la matriz de varianzas y covarianzas de los momentos observados.

${ }^{13}$ Véase el apéndice A.5 para una discusión más detallada de la implementación práctica del método de momentos simulados.
} 
El primero de ellos es la correlación serial de la tasa de inversión $\left(\operatorname{corr}\left(i, i_{-1}\right)\right)$. El segundo momento consiste en la correlación entre la tasa de inversión y (el logaritmo de) los shocks de rentabilidad $(\operatorname{corr}(i, a))$. Adicionalmente, con el objetivo de capturar tanto la asimetría como la amplia cola hacia la derecha de la distribución de la tasa de inversión a nivel de la planta, incluimos como momentos a la fracción de observaciones con tasa de inversión superior al $20 \%$ (prop > 0.20) y a la fracción de observaciones con tasa de inversión menor al $-5 \%$ (prop $<-0.05)$.

La tabla 5 presenta los parámetros estimados para los tres componentes de los costos de ajuste del capital, junto con los errores estándar de las estimaciones. ${ }^{14}$ Adicionalmente, la tabla reporta tanto los momentos observados como simulados cuya distancia entre los mismos es la que busca ser minimizada, como así también una medida de la bondad de ajuste del modelo $\left(\Gamma\left(\Theta_{K}\right)\right)$. La parametrización utilizada para resolver el modelo y generar una versión simulada de los datos se corresponde con la descrita en la tabla 4.

Los parámetros de costo de ajuste estimados implican grandes costos fijos e irreversibilidades, junto con moderados costos cuadráticos. Todos los parámetros estimados resultan significativos estadísticamente para un nivel de confianza del $5 \%$. Los costos fijos estimados son iguales a $f=0.1001$. Esto indica la presencia de grandes costos fijos en el ajuste del capital, implicando que los costos fijos de ajustar el stock de capital representan aproximadamente el $10 \%$ del stock de capital promedio a nivel de la planta. El coeficiente estimado para el parámetro asociado al costo de ajuste cuadrático $(\gamma)$ es igual a 0.0315. Considerando la función de costos de ajuste cuadrático (2-5) junto a un nivel de estado estacionario para la tasa de inversión igual a la tasa de depreciación ( $I / K=\delta=9.91 \%$ ), el parámetro estimado representa un costo de ajuste relativo al capital promedio de la planta de $0.0155 \%$. Finalmente, las estimaciones obtenidas para el parámetro asociado a los costos de transacción $\left(p_{s}=0.8836\right)$ indican que la venta de bienes de capital conllevan una pérdida de aproximadamente el $12 \%$ del precio original de compra.

El gráfico 2 presenta las reglas de decisión óptimas (para un shock de rentabilidad dado y arbitrario) de diferentes versiones restringidas del modelo estimado. Para cada una de estas versiones, el modelo dinámico es resuelto y las reglas de decisión son computadas en base a los parámetros estimados discutidos en el párrafo precedente. El gráfico también presenta la regla de decisión para el modelo sin fricciones (No CAC), donde no existen costos por ajustar el stock de capital. ${ }^{15}$ Observamos que tanto los

\footnotetext{
${ }^{14}$ El apéndice A.5.3 describe la metodología utilizada para computar los errores estándar.

15 En este caso, el vector de parámetros asociado a la función de costos de ajuste en el capital es
} 
costos fijos como las irreversibilidades parciales generan una región de inacción, cancelando la respuesta de la inversión. Esta estructura de costos produce también episodios de gran inversión y desinversión (un rasgo observado en los datos). Es interesante notar que, sin embargo, la decisión óptima de inversión bajo el modelo con costos fijos difiere a la obtenida bajo el modelo con irreversibilidades parciales. En particular, ajustes más grandes e infrecuentes en el stock de capital son necesarios para compensar los costos fijos. Finalmente, los costos de ajuste cuadráticos actúan reduciendo la respuesta de la inversión, y creando correlación serial positiva en las tasas de inversión. El gráfico 3 presenta las reglas de decisión para el modelo irrestricto, incorporando los tres tipos de costos en la función de ajuste.

Las estimaciones obtenidas para los parámetros de costos son comparables con las obtenidas por Cooper y Haltiwanger (2006). Tanto la metodología seguida en el presente trabajo como la naturaleza de los datos (datos a nivel de la planta) son similares a los utilizados por los autores mencionados. Cooper y Haltiwanger (2006) estiman menores costos fijos (0.039) e irreversibilidades parciales (0.975); por otro lado, estiman un valor levemente superior (0.049) para el parámetro asociado al costo de ajuste cuadrático. Otros trabajos han estimado costos de ajuste del capital siguiendo una metodología similar a la presentada en la presente sección. Específicamente, los trabajos de Bloom (2009) y Bond et al. (2008) estiman pérdidas asociadas a la reventa de capital del orden del $47 \%$ y $16.9 \%$, respectivamente; lo que implica costos de transacción más altos que los estimados en el presente estudio. Asimismo, ambos trabajos obtienen valores más elevados que los que estimamos para la muestra de empresas argentinas para el parámetro asociado a los costos de ajustes cuadráticos (Bloom (2009) estima dicho parámetro en 2.056, mientras que Bond et al. (2008) obtiene un valor de 1.985 para el mismo). Finalmente, estos trabajos estiman los costos fijos en términos de las ventas anualizadas (en lugar de hacerlo en términos del stock de capital promedio de la planta). Las magnitudes obtenidas por los mismos varían entre el $0.3 \%$ (Bond et al. (2008)) y el $1.3 \%$ de las ventas anuales (Bloom (2009)). ${ }^{16}$

${ }^{16}$ Los resultados presentados para estos dos trabajos son aquellos vinculados a la estimación bajo el supuesto de una planta por firma. Ambos trabajos estiman los parámetros de costos a nivel de la firma, realizando supuestos sobre el número de plantas (con el objetivo de agregar en la dimensión cross-section). Esta agregación cross-section tiene implicancias sobre la identificación de parámetros dado que la misma suaviza el comportamiento de la inversión. Por este motivo, se elige reportar la especificación más cercana a la utilizada en el presente trabajo. Cabe destacar que a la hora de comparar los resultados obtenidos en el presente estudio con los hallados por Bloom (2009) y Bond et al. (2008) deben tenerse en cuenta las diferencias en las especificaciones presentadas por los autores mencionados, ya que ambos identifican en sus estimaciones parámetros adicionales a los de costos de ajuste en el capital.
} 


\section{Costos de ajuste del capital y mercados laborales: efectos de un shock de rentabilidad}

Haciendo uso del modelo teórico planteado en la sección 2, en esta sección se estudiarán las implicancias de un shock negativo en la rentabilidad media de las plantas. Específicamente, analizaremos los efectos potenciales del shock de rentabilidad considerando sus implicancias sobre el nivel de capital agregado de la economía, el nivel de salarios y el nivel agregado de empleo. Para ello, se asumirá que todas las plantas enfrentan costos por ajustar su stock de capital, con una tecnología de costos de ajuste del capital descripta por los parámetros presentados en la tabla 5. Asimismo, los parámetros tecnológicos, de demanda y aquellos asociados a el proceso estocástico se corresponden con los estimados en el trabajo y presentados en la tabla 4.

A fines de evaluar los efectos del shock de rentabilidad sobre el nivel de empleo agregado de la economía y los salarios, es necesario especificar el comportamiento del mercado de trabajo. Se asumirá que el mercado de trabajo es competitivo. Como consecuencia, los salarios se determinan por la interacción entre la oferta y demanda de trabajo. Consideremos el problema de decisión enfrentado por una firma que debe elegir su demanda de trabajo y posee variables de estado (K,A). Dado que las firmas ajustan sus factores variables sin costo alguno, la demanda de trabajo viene dada por la siguiente ecuación:

$$
L_{i t}\left(K_{i t}, A_{i t} ; w_{t}\right)=\frac{\phi_{l}}{\left(1-\phi_{l}-\phi_{m}\right)}\left(w_{t}\right)^{\frac{1-\phi_{m}}{\phi_{m}+\phi_{l}-1}} A_{i t} K_{i t}^{\theta}
$$

donde $A_{i t}$ incorpora variaciones en $z_{t}, D_{i t}$, y $\widetilde{A_{i t}} \cdot{ }^{17}$

El empleo agregado en el período $t$ vendrá dado por:

$$
N_{t}=\int L_{i t}\left(K_{i t}, A_{i t} ; w_{t}\right) \mu(d K \times d A)
$$

donde $\mu(d K \times d A)$ representa la distribución conjunta cross-section de $(K, A)$, la cual satisface en el largo plazo estacionariedad:

$$
\mu(\Lambda \times Z)=\int 1_{g(K, A ; w) \in \Lambda} \mathcal{Q}(A, Z) \mu(d K \times d A)
$$

\footnotetext{
17 Notar que, mientras en el modelo presentado en la sección 2 los salarios son estocásticos, en este caso es necesario tratar a los mismos como determinísticos a fines de analizar los efectos del shock sobre los salarios. Para ello es necesario excluirlos de A. De esta forma, $A_{i t}=\left(1-\phi_{l_{j}}-\right.$ $\left.\phi_{m_{j}}\right) D_{i t}^{1 / \varepsilon} \widetilde{A}_{i t}^{\frac{1-1 / \varepsilon}{1-\phi_{l}-\phi_{m}}}\left(1 / \phi_{l_{j}}\right)^{\frac{\phi_{l_{j}}}{\phi_{l_{j}+\phi_{m_{j}}-1}}}\left(z_{t} / \phi_{m_{j}}\right)^{\frac{\phi_{m_{j}}}{\phi_{l_{j}}+\phi_{m_{j}}-1}}$
} 
para cualquier conjunto $\Lambda$ y $Z$ (donde $\mathcal{Q}($.$) representa la función de transición del$ proceso de Markov $\left.A_{i t}\right)$.

Trabajaremos bajo dos supuestos alternativos de funcionamiento del mercado de trabajo. En primer lugar, asumiremos que la oferta de trabajadores está fija. Esto implica que los efectos del shock generarán dinámicas sobre el salario de la economía. En segundo lugar, se trabajará bajo el supuesto de oferta de trabajo infinitamente elástica al salario nominal $\bar{w}$. En este caso, los efectos del shock tendrán consecuencias sobre el nivel de empleo agregado de la economía.

Se considerará una reducción en la rentabilidad media de las empresas del $50 \%$. Dicho shock ocurre al inicio del período $t=0$. En los períodos previos a la ocurrencia del shock, la economía se encuentra en un estado estacionario.

La reducción en la rentabilidad media de las firmas genera diferentes efectos sobre el mercado laboral. Estos efectos dependerán de los supuestos asumidos sobre el comportamiento del mercado de trabajo. En el modelo con oferta de trabajo infinitamente elástica se espera que los efectos del shock repercutan con una mayor fuerza sobre el capital agregado de la economía, dada la complementariedad tecnológica de factores y la imposibilidad de traspasar pérdidas de rentabilidad al salario. Por el contrario, si la oferta de trabajo está fija, el shock adverso generará una reducción en el salario. Es interesante notar que estas reducciones en el salario morigeran los efectos iniciales y adversos del shock, dado que mejoran las condiciones de rentabilidad de las firmas.

El gráfico 4 muestra la evolución del empleo agregado en la economía luego de un shock de rentabilidad adverso (del $50 \%$ ) sobre las firmas, ocurrido en el período 0 . En este caso la oferta de trabajo es infinitamente elástica. El empleo agregado es normalizado a 1 al inicio con el objetivo de interpretar con mayor facilidad la caída porcentual en la variable. Los resultados implican un gran efecto del shock sobre el mercado de trabajo. En particular, el nivel de empleo cae aproximadamente un $60 \%$ entre el estado estacionario inicial y el final. Asimismo, se observa que el ajuste es lento. La economía tarda 9 años en alcanzar el nuevo estado estacionario.

El gráfico 5 presenta los resultados sobre el mercado de trabajo bajo el supuesto de oferta de trabajo fija. Al igual que en el caso anterior, se observa que el shock de rentabilidad posee efectos de considerable magnitud sobre el mercado laboral. Particularmente, el salario de equilibrio cae en aproximadamente $40 \%$ respecto a su valor inicial. Asimismo, la economía demora 5 años en alcanzar su nuevo estado estacionario.

La evolución del capital agregado se exhibe en el gráfico 6. En el mismo, se presenta la evolución del capital bajo los dos supuestos alternativos de funcionamiento del mercado de trabajo. Es interesante notar, que los supuestos de funcionamiento del mercado 
laboral afectan sustancialmente a la dinámica de ajuste del capital. Específicamente, observamos que la magnitud del ajuste entre estados estacionarios es un $20 \%$ mayor en el modelo que supone la existencia de una oferta de trabajo infinitamente elástica con respecto al modelo con oferta de trabajo fija. Este resultado, resalta la importancia de el conocimiento de los costos y fricciones de movilidad que enfrenta el factor trabajo a efectos de evaluar con mayor precisión las consecuencias de una política o shocks sobre la economía. El trabajo de Bet et al. (2011) constituye un aporte en esta dirección para el caso de Argentina, al incorporar y cuantificar, en un modelo con costos de ajuste de capital, fricciones del mercado laboral.

Con el objetivo de evaluar la robustez de las simulaciones a diferentes magnitudes del shock, los gráficos 7, 8, 9 y 10 presentan la evolución del empleo, salarios y capital agregado para shocks sobre la rentabilidad media de las firmas del 30\%, $50 \%$ y $70 \%$. Los resultados indican que shocks de mayores magnitudes no sólo implican mayores efectos sobre las variables, sino que también efectos más persistentes sobre las mismas.

\subsection{Movilidad del capital y costos de ajuste}

En esta sección se analizan los resultados predichos por el modelo bajo diferentes supuestos de movilidad del capital. En particular, se comparan los resultados del modelo con costos de ajuste en el capital, con los obtenidos asumiendo capital fijo o ausencia de costos de ajuste.

Los gráficos 11, 12 y 13 exhiben la evolución del empleo, salarios y capital agregado bajo diferentes supuestos de movilidad del capital. En primer lugar, se observa que la movilidad del capital magnifica los efectos del shock sobre el mercado de trabajo. Esto implica mayores cambios en el nivel de empleo (bajo el supuesto de oferta de trabajo infinitamente elástica) y en el nivel de salarios (bajo el supuesto de oferta de trabajo fija). En los modelos con capital móvil, la caída en el nivel de empleo agregado es aproximadamente un $10 \%$ mayor que la caída observada en el modelo con capital fijo. Adicionalmente, las simulaciones contrafácticas realizadas indican que la reducción en los salarios para los modelos con capital móvil es aproximadamente un $5 \%$ mayor que la reducción estimada en el modelo con capital fijo.

En segundo lugar, los costos de ajuste del capital constituyen un factor importante en el ajuste lento del mercado de trabajo. Los gráficos 11, 12 y 13 muestran que aunque los cambios en las magnitudes de largo plazo son los mismos en los modelos con ajuste costoso y no costoso en el capital, las dinámicas son mucho más lentas en el primer caso. 
Finalmente, el gráfico 13 muestra que, si bien existen dinámicas de ajuste del capital en el modelo sin fricciones, la mayor parte de la transición se realiza en el primer año. Por otro lado, el ajuste del capital en el modelo con fricciones es lento, tomándole a las firmas 5 o 9 años completar el ajuste en función de los supuestos realizados sobre el mercado de trabajo.

\section{Conclusiones}

El presente trabajo presenta un modelo dinámico estructural de demanda por inversión con firmas heterogéneas y costos de ajuste del capital. En el modelo, la toma de decisiones de inversión se realiza a nivel de la firma. Esta característica del modelo permite analizar la naturaleza de los costos de ajuste del capital a nivel micro, y las implicancias que dichas fricciones y la movilidad de capital imponen sobre el ajuste de factores ante la presencia de shocks en la economía.

Se estiman los parámetros de interés utilizando datos para un panel de 568 plantas manufactureras argentinas, abarcando el período 1994-2001. Las estimaciones realizadas permiten recuperar una medida de las fricciones enfrentadas por las firmas para ajustar su stock de capital.

Los resultados hallados para los costos de ajuste del capital son consistentes con los obtenidos por otros autores siguiendo metodologías de estimación similares. Nuestras estimaciones destacan la relevancia de las convexidades, no-convexidades e irreversibilidades en la estructura de costos de ajuste a nivel de las plantas. En particular encontramos que existen grandes costos fijos e irreversibilidades, junto con moderados costos cuadráticos.

Los parámetros estimados son utilizados luego en simulaciones contrafácticas para analizar los efectos sobre el mercado de trabajo de una caída en la rentabilidad media de las firmas. Los resultados sugieren una gran respuesta del mercado laboral al shock. Adicionalmente, la transición hacia el nuevo estado estacionario es lenta, completándose el ajuste en varios años.

Finalmente, los ejercicios de simulación realizados resaltan la importancia no sólo de modelar la movilidad del capital, sino también la de considerar y estimar las fricciones asociadas al mismo, con el objetivo de evaluar los efectos de políticas o shocks sobre la economía. En particular, la movilidad y costos de ajuste del capital no sólo afectan la velocidad de ajuste de la economía en respuesta a un shock, sino que también la 
magnitud del impacto sobre la asignación y remuneración a los factores en el corto y largo plazo. 


\section{A. APÉNDICE}

\section{A.1. Maximización de beneficios a corto plazo}

El problema de maximización de beneficios a corto plazo enfrentado por la firma consiste en:

$$
\begin{aligned}
& \pi_{i t}\left(K_{i t}, A_{i t}\right)=\operatorname{máx}_{L \geq 0 ; M \geq 0}\left\{P_{i t} Q_{i t}-w_{t} L_{i t}-z_{t} M_{i t}\right\} \\
& \pi_{i t}\left(K_{i t}, A_{i t}\right)=\operatorname{máx}_{L \geq 0 ; M \geq 0}\left\{D_{i t}^{1 / \varepsilon} \widetilde{A}_{i t}^{\frac{1-1 / \varepsilon}{1-\phi_{l}-\phi_{m}}} K_{i t}^{\phi_{k}} L_{i t}^{\phi_{l}} M_{i t}^{\phi_{l}}-w_{t} L_{i t}-z_{t} M_{i t}\right\}
\end{aligned}
$$

donde $\phi_{j}=\alpha_{j}(1-1 / \varepsilon)$ para $j=l, m, k$.

De las condiciones de primer orden del problema es posible obtener la demanda indirecta de trabajo $\left(L^{*}\left(K_{i t}, A_{i t}\right)\right)$ y materiales $\left(M^{*}\left(K_{i t}, A_{i t}\right)\right)$ en términos de las variables de estado del problema. Reemplazando las mismas en (A-2) se obtiene la función de beneficios operativos de la firma en términos de las variables de estado:

$$
\pi_{i t}\left(K_{i t}, A_{i t}\right)=A_{i t} K_{i t}^{\theta}
$$

donde,

$$
\theta=\frac{\phi_{k}}{1-\phi_{l}-\phi_{m}}
$$

$\mathrm{y}$

$$
A_{i t}=\left(1-\phi_{l_{j}}-\phi_{m_{j}}\right) D_{i t}^{1 / \varepsilon} \widetilde{A}_{i t}^{\frac{1-1 / \varepsilon}{1-\phi_{l}-\phi_{m}}}\left(\frac{w_{t}}{\phi_{l_{j}}}\right)^{\frac{\phi_{l_{j}}}{\phi_{l_{j}+\phi_{m_{j}}-1}}}\left(\frac{z_{t}}{\phi_{m_{j}}}\right)^{\frac{\phi_{m_{j}}}{\phi_{l_{j}}+\phi_{m_{j}}-1}}
$$

\section{A.2. La relación entre ingresos, costos y elasticidad de demanda}

El problema de maximización de beneficios a corto plazo de la firma puede ser expresado en forma alternativa como:

$$
\pi_{i t}\left(Q_{i t}\right)=\operatorname{máx}_{Q \geq 0}\left\{P_{i t}\left(Q_{i t}\right) Q_{i t}-c_{i t}\left(Q_{i t}\right)\right\}
$$


La condición de primer orden del problema está dada por:

$$
\begin{aligned}
P_{i t}\left(Q_{i t}\right)+P_{i t}^{\prime}\left(Q_{i t}\right) Q_{i t}-c_{i t}^{\prime}\left(Q_{i t}\right) & =0 \\
P_{i t}\left(Q_{i t}\right)-\frac{1}{\varepsilon} P_{i t}-c_{i t}^{\prime}\left(Q_{i t}\right) & =0
\end{aligned}
$$

donde la ecuación (A-8) se deriva de los supuestos realizados sobre las demandas enfrentadas por las firmas.

Reordenando la ecuación (A-8) se obtiene la ecuación para el markup sobre costos marginales de la firma:

$$
P_{i t}\left(Q_{i t}\right)\left(1-\frac{1}{\varepsilon}\right)=c_{i t}^{\prime}\left(Q_{i t}\right)
$$

Notar que es posible derivar la función de costos indirecta $c_{i t}\left(Q_{i t}, w_{t}, z_{t}\right)$ asociada al problema de optimización (A-6) luego de obtener las demandas óptimas para los factores variables:

$$
\begin{aligned}
& c_{i t}\left(Q_{i t}, w_{t}, z_{t}\right)=w_{t} L_{i t}^{*}\left(Q_{i t}, w_{t}, z_{t}\right)+z_{t} M_{i t}^{*}\left(Q_{i t}, w_{t}, z_{t}\right) \\
& c_{i t}\left(Q_{i t}, w_{t}, z_{t}\right)=\varpi\left(w_{t}, z_{t}, \widetilde{A_{i t}}, K_{i t}\right) Q_{i t}^{\frac{1}{\alpha_{l}+\alpha_{m}}}
\end{aligned}
$$

donde $\varpi\left(w_{t}, z_{t}, \widetilde{A_{i t}}, K_{i t}\right)$ es una función de $w_{t}, z_{t}, \widetilde{A_{i t}} \mathrm{y} K_{i t}$. De esta forma, el costo marginal de producción $\left(c_{i t}^{\prime}\right)$ vendrá dado por:

$$
c_{i t}^{\prime}=\frac{\varpi\left(w_{t}, z_{t}, \widetilde{A_{i t}}, K_{i t}\right) Q_{i t}^{\frac{1}{\alpha_{l}+\alpha_{m}}}}{\left(\alpha_{l}+\alpha_{m}\right) Q_{i t}}
$$

Asimismo, notemos que:

$$
c_{i t}^{\prime} Q_{i t}=\frac{c_{i t}}{\alpha_{l}+\alpha_{m}}
$$

Luego de introducir la ecuación (A-12) dentro de la ecuación (A-9) y multiplicar ambos lados de esta última por $Q_{i t}$, obtenemos la ecuación (4-1) que relaciona los ingresos y costos de la firma con la elasticidad de demanda:

$$
\frac{R_{i t}-c_{i t} /\left(\alpha_{l}+\alpha_{m}\right)}{R_{i t}}=\frac{1}{\varepsilon}
$$

donde $R_{i t}=P_{i t} Q_{i t}$ representa los ingresos por ventas de la firma. 


\section{A.3. Construcción y Medición de Variables}

Esta sección describe la metodología utilizada para la construcción de la serie de stock de capital real e inversión real neta a nivel de la planta. Asimismo, se discuten aspectos vinculados con la definición de ciertas variables utilizadas en el trabajo y su medición.

Todas las variables monetarias fueron llevadas a magnitudes reales deflactando las series mediante el Índice de Precios Internos Básicos al por Mayor (IPIB). Las variables inversión, stock de capital y consumo intermedio fueron deflactadas utilizando el nivel general del índice. Por último, el valor bruto de producción, los ingresos por ventas y los beneficios fueron deflactados utilizando la desagregación a cuatro dígitos del índice, la cual permite deflactar las variables por ramas de actividad específica a la planta.

A fines de concretar el análisis empírico es necesario generar una medida del stock de capital real a nivel de la planta. El procedimiento comúnmente utilizado consiste en estimar un valor inicial para el stock de capital real de la planta para algún año y luego generar la serie de stock de capital utilizando el método de inventarios permanente:

$$
K_{i, t+1}=(1-\delta) K_{i t}+I_{i t}
$$

donde $I_{i t}$ representa la inversión real neta, $K_{i t}$ el stock de capital real, y $\delta$ la tasa de depreciación del capital.

La inversión real neta se define como:

$$
I_{i t}=G_{i t}-S_{i t}
$$

donde $G_{i t}$ representa el gasto real bruto en bienes de capital, y $S_{i t}$ las ventas reales brutas.

Utilizamos las ecuaciones (A-15) y (A-16) para generar la serie de stock de capital medido en términos reales. Dado que nuestra base de datos no contiene información acerca del valor de libros del capital, aproximamos el stock de capital inicial de la planta como el cociente entre el valor promedio del monto de depreciación de capital declarado por la planta (a lo largo de los años) y la tasa de depreciación estimada para la industria. Deflactamos dicha estimación del stock de capital inicial por el nivel general del IPIB a fines de obtener una estimación del stock de capital inicial en términos reales. Como no contamos con información de tasas de depreciación del capital para la industria manufacturera Argentina utilizamos como aproximación las estimaciones sectoriales realizadas por el Departamento de Análisis Económicos (Bureau of Economic Analysis 
(BEA)) de los Estados Unidos para la industria manufacturera de dicho país. ${ }^{18}$ Las tasas de depreciación utilizadas incluyen tanto el componente asociado a la pérdida de eficiencia del bien por el uso y desgaste del mismo, como el asociado a los retiros de capital por descarte o desecho del bien (lo cual puede estar asociado a la obsolescencia o agotamiento del bien en cuestión). Por lo tanto, no necesitaremos estimar patrones de retiros de capital por industria.

\section{A.4. Estimación Función Producción}

El presente apéndice describe la metodología de estimación de los parámetros asociados a la función de producción. Como fue mencionado en la sección 4, la estimación de la curvatura de la función de producción se efectúa en una etapa previa a la estimación de los parámetros asociados a los costos de ajuste de la firma. En particular, estimamos dichos parámetros mediante la técnica desarrollada por Olley y Pakes (1996), que permite controlar el problema de simultaneidad presente en esta clase de estimaciones utilizando la inversión como proxy de los shocks de productividad inobservables que varían en el tiempo.

Estimamos la siguiente variante de la ecuación (2-1):

$$
\begin{array}{r}
q_{i t}=\alpha_{0}+\alpha_{k} k_{i t}+\alpha_{l} l_{i t}+\alpha_{m} m_{i t}+u_{i t} \\
u_{i t}=\varkappa_{i t}+\eta_{i t}
\end{array}
$$

donde $q_{i t}$ es el logaritmo del valor de producción de la firma $i$ en el período $t$ (medido a partir del valor bruto de producción); $l_{i t}$ y $m_{i t}$ representan el logaritmo de los insumos variables trabajo y consumo intermedio, respectivamente; $k_{i t}$ es el logaritmo del capital (variable de estado); y $u_{i t}$ es un término de error compuesto por un shock de productividad $\varkappa_{i t}$ observado sólo por la firma y un shock de productividad $\eta_{i t}$ inesperado e inobservado. ${ }^{19}$ Mientras que $\eta_{i t}$ no tiene efectos en las decisiones de la firma, $\varkappa_{i t}$ es una variable de estado que afecta el proceso de decisión de la firma. El supuesto de que $\varkappa_{i t}$ es sólo observado por la firma implica que los insumos estarán correlacionados con los shocks de productividad. En particular, Olley y Pakes (1996) asumen que la

\footnotetext{
18 Para una discusión detallada de la metodología de cálculo de las tasas de depreciación sectoriales estimadas por la BEA véase Fraumeni (1997).

19 En la práctica, se computó el nivel del factor trabajo como el total de obreros asalariados de la planta ponderados por el ratio del salario promedio pagado por la planta al salario promedio pagado por la industria. Esta definición permite controlar por los diferenciales de productividad en el trabajo empleado en la planta relativa a la del promedio de la industria. Asimismo, medimos tanto el stock de capital como el consumo intermedio y el valor bruto de producción en términos reales.
} 
productividad futura es estrictamente creciente respecto a la productividad corriente $\left(\varkappa_{i t}\right)$, de modo tal que las firmas que observan un shock de productividad positivo invertirán más en dicho período (independientemente del stock de capital corriente). Este supuesto permite escribir a $\varkappa_{i t}$ cómo una función (desconocida) de la inversión $\left(i_{i t}\right)$ y el capital $\left(k_{i t}\right):^{20}$

$$
\varkappa_{i t}=h\left(i_{i t}, k_{i t}\right)
$$

Al introducir las ecuaciones (A-18) y (A-19) dentro de (A-17) obtenemos:

$$
\begin{array}{r}
q_{i t}=\alpha_{l} l_{i t}+\alpha_{m} m_{i t}+\psi\left(i_{i t}, k_{i t}\right)+\eta_{i t} \\
\psi\left(i_{i t}, k_{i t}\right)=\alpha_{0}+\alpha_{k} k_{i t}+h\left(i_{i t}, k_{i t}\right)
\end{array}
$$

Luego de aproximar $\psi($.$) mediante un polinomio de tercer orden en el capital y la$ inversión, estimamos la ecuación (A-20) utilizando el método de mínimos cuadrados ordinarios. Dado que $\psi($.$) controla por la productividad no observada, obtenemos es-$ timaciones consistentes para los factores variables (trabajo y consumo intermedio).

En una segunda etapa, con el objetivo de recuperar el parámetro $\alpha_{k}$, estimamos la siguiente ecuación:

$$
q_{i t}-\widehat{\alpha_{l}} l_{i t}-\widehat{\alpha_{m}} m_{i t}=\alpha_{k} k_{i t}+\varphi\left(\widehat{\psi}_{t-1}-\alpha_{k} k_{i, t-1}\right)+\xi_{i t}+\eta_{i t}
$$

aproximando la función $\varphi($.$) mediante un polinomio de cuarto orden en \widehat{\psi}_{t-1}-\alpha_{k} k_{i, t-1}$. Finalmente computamos los errores estándar mediante bootstrap.

\section{A.5. Métodos Numéricos}

Este apéndice describe algunas técnicas numéricas utilizadas para resolver el problema dinámico intertemporal de la firma y estimar los parámetros de costos de ajuste del capital a través del método de momentos simulados (MMS).

\section{A.5.1. Iteración sobre la función de Valor}

La función de valor es utilizada en diferentes etapas del trabajo. En una primera etapa, con el objetivo de estimar los parámetros de costos de ajuste del capital, es

\footnotetext{
20 El supuesto realizado por Olley y Pakes (1996) implica que la inversión se restrinja a valores estrictamente positivos. Esto impone ciertas restricciones en nuestras estimaciones, implicando que tanto los valores negativos como nulos de la inversión sean descartados en las mismas.
} 
necesario resolver el modelo dinámico y obtener las reglas de decisión óptimas de la firma para generar una versión artificial de los datos. En una segunda etapa, las reglas de decisión óptimas son utilizadas para simular los efectos de un shock de rentabilidad sobre el ajuste del capital a nivel de las firmas. En ambos casos, el método numérico utilizado para resolver la función de valor (2-10) es el mismo. El método implica, en primer lugar, discretizar el espacio de las variables del problema, para lo cual es necesario generar una grilla de puntos en el espacio de las variables $(K, I, A)$. Las variables $K$ e $I$ son discretizadas mediante una grilla de 400 elementos. Adicionalmente, dado que el problema requiere tomar la esperanza condicional sobre $A$, se utiliza la metodología propuesta por Tauchen y Hussey (1991) para discretizar el espacio del proceso continuo de Markov. El espacio para esta variable es discretizado con una grilla de 22 elementos. De esta forma, el espacio de variables $(K, I, A)$ toma las dimensiones (400x400x22).

En una segunda etapa, se resuelve la función de valor iterando sobre la misma. Para ello, es necesario elegir primero un valor arbitrario inicial para dicha función con el objetivo de dar inicio a las iteraciones; el valor hallado para la función de valor es luego utilizado en la próxima iteración. Con el objetivo de garantizar la convergencia en las reglas de decisión óptimas de la firma, se establece como criterio de convergencia una diferencia porcentual máxima entre dos funciones de valor correspondientes a iteraciones consecutivas menor al $0.01 \%$. En la práctica, las funciones de valor convergen en forma más lenta que las reglas de decisión asociadas a las mismas. ${ }^{21}$

\section{A.5.2. Algoritmo de Optimización: Simulated Annealing}

Con el objetivo de minimizar la función de pérdida (4-5) utilizamos un algoritmo de búsqueda denominado simulated annealing. Dicho algoritmo es programado para buscar en el espacio de parámetros de la siguiente forma: (i) en primer lugar, para un vector inicial (arbitrario) de parámetros, la función de valor es obtenida y las reglas de decisión asociadas a las mismas son utilizadas para generar una versión simulada de los datos; (ii) luego, utilizando dichos datos, se computan un conjunto de momentos junto con la función de pérdida (4-5) asociada a los mismos; (iii) en una segunda iteración, se propone un nuevo vector de parámetros con el objetivo de computar nuevamente la función de pérdida; (iv) a partir de la tercera iteración en adelante, los nuevos

\footnotetext{
${ }^{21}$ Ciertas condiciones de regularidad aseguran que la solución sea única: a) una función de utilidad aditivamente separable en el tiempo; b) una función de utilidad para un período acotada; c) matrices de transición de Markov invariantes en el tiempo; y d) un factor de descuento $\beta \in(0,1)$. Para una discusión en detalle véase Rust (1996).
} 
vectores de parámetros son propuestos sobre la base del mejor vector propuesto hasta el momento (aquel que momentáneamente minimiza la función de pérdida).

El número total de iteraciones realizadas fue establecido en 6000. Para las primeras 1500 iteraciones, el nuevo vector de parámetros surge como la suma de el vector de parámetros que minimiza la función de pérdida y un componente aleatorio. A partir de la iteración 1500 en adelante, se incluyó un componente direccional en el algoritmo de búsqueda. ${ }^{22} \mathrm{El}$ algoritmo también fue programado de modo tal que la varianza del parámetro caiga a medida que se avanza en el número de iteraciones, permitiendo de esta forma refinar la búsqueda de los parámetros a ser estimados alrededor del óptimo global. Finalmente, dado que el estimador utiliza números aleatorios y depende de las condiciones iniciales asumidas, se realizaron diferentes pruebas variando las seeds y las condiciones iniciales con el objetivo de garantizar la convergencia de los parámetros alrededor del mínimo global. En todos los casos estas pruebas fueron satisfactorias.

\section{A.5.3. Errores Estándar (MMS)}

Para generar los errores estándar es necesario computar la matriz de varianzas y covarianzas del estimador. Dada la elección eficiente de la matriz de ponderación $W$, el estimador del MMS es asintóticamente normal para $N_{o}$ fijo cuando $N_{s} \rightarrow \infty$ :

$$
\sqrt{N}\left(\widehat{\Theta}-\Theta^{*}\right) \rightarrow N(0, V)
$$

donde,

$$
V=\left(J^{\prime}(1+1 / n)^{-1} \Omega^{-1} J\right)^{-1}
$$

con

$$
J=\frac{\partial \Psi^{S}(\widehat{\Theta})}{\partial \Theta}=\left[\begin{array}{lll}
\frac{\partial \Psi_{1}^{S}(\widehat{\Theta})}{\partial \Theta_{1}} & \frac{\partial \Psi_{1}^{S}(\widehat{\Theta})}{\partial \Theta_{2}} & \frac{\partial \Psi_{1}^{S}(\widehat{\Theta})}{\partial \Theta_{3}} \\
\frac{\partial \Psi_{2}^{S}(\widehat{\Theta})}{\partial \Theta_{1}} & \frac{\partial \Psi_{2}^{S}(\widehat{\Theta})}{\partial \Theta_{2}} & \frac{\partial \Psi_{2}^{S}(\widehat{\Theta})}{\partial \Theta_{3}} \\
\frac{\partial \Psi_{3}^{S}(\widehat{\Theta})}{\partial \Theta_{1}} & \frac{\partial \Psi_{3}^{S}(\widehat{\Theta})}{\partial \Theta_{2}} & \frac{\partial \Psi_{3}^{S}(\widehat{\Theta})}{\partial \Theta_{3}} \\
\frac{\partial \Psi_{4}^{S}(\widehat{\Theta})}{\partial \Theta_{1}} & \frac{\partial \Psi_{4}^{S}(\widehat{\Theta})}{\partial \Theta_{2}} & \frac{\partial \Psi_{4}^{S}(\widehat{\Theta})}{\partial \Theta_{3}}
\end{array}\right]
$$

La matriz Jacobiana $J$ debe ser computada numéricamente. Un problema práctico

\footnotetext{
${ }^{22}$ Inicialmente se programó el algoritmo incluyendo el componente direccional en las 6000 iteraciones. Esto generó inconvenientes en la búsqueda, vinculados con la imposibilidad de poder superar mínimos locales. Por esta razón, se optó por excluir el componente direccional en el algoritmo de búsqueda en las primeras 1500 iteraciones.
} 
asociado a dicha estimación radica en el hecho de que el valor de la derivada numérica, definida como $\frac{\partial \Psi^{S}(\widehat{\Theta})}{\partial \Theta}=\frac{\Psi^{S}(\widehat{\Theta}+\epsilon)-\Psi^{S}(\widehat{\Theta})}{\epsilon}$, es sensible al valor de $\epsilon$ en el cual la derivada es evaluada. Como menciona Bloom (2009), esta clase de problemas es común en métodos numéricos con datos simulados que utilizan funciones con potenciales discontinuidades (las cuales pueden surgir, por ejemplo, producto de la discretización de las variables de estado del problema). A fines de resolver este inconveniente, calculamos las derivadas numéricas siguiendo una estrategia similar a la utilizada por Bloom (2009). En particular, calculamos cuatro valores para las derivadas con valores de $\epsilon$ iguales a $+1 \%,+2.5 \%,+5 \%$ y $-1 \%$ del valor del parámetro estimado. Luego de ello, tomamos la mediana de las derivadas numéricas computadas. Este procedimiento contribuye a que las derivadas estimadas sean robustas a outliers producto de discontinuidades en las funciones bajo análisis. 


\section{Referencias}

Abel, Andrew B and Janice C Eberly, "A Unified Model of Investment under Uncertainty," American Economic Review, December 1994, 84 (5), 1369-84.

_ and _ , "Optimal Investment with Costly Reversibility," Review of Economic Studies, October 1996, 63 (4), 581-93.

Bet, Germán, Irene Brambilla, y Guido Porto, "Trade Reforms, Wages and Employment with Labor Mobility and Capital Adjustment Costs," Working Paper, Universidad Nacional de La Plata 2011.

Bloom, Nicholas, "The Impact of Uncertainty Shocks," Econometrica, 05 2009, 7 r (3), 623-685.

Bond, Stephen R., Mans Soderbom, y Guiying Wu, "A Structural Estimation for the Effects of Uncertainty on Capital Accumulation with Heterogeneous Firms," 2008.

Caballero, Ricardo J. and Eduardo Engel, "Adjustment is Much Slower than You Think," NBER Working Papers 9898, National Bureau of Economic Research, Inc August 2003.

_ and Eduardo M. R. A. Engel, "Explaining Investment Dynamics in U.S. Manufacturing: A Generalized (S,s) Approach," Econometrica, July 1999, 67 (4), 783-826.

Caballero, Ricardo J, Eduardo M R A Engel, y John Haltiwanger, "PlantLevel Adjustment and Aggregate Investment Dynamics," Brookings Papers on Economic Activity, 1995, (2), 1-54.

Cooper, Russel W., John C. Haltiwanger, y Jonathan Willis, "Dynamics of Labor Demand: Evidence from Plant-level Observations and Aggregate Implications," NBER Working Papers 10297, National Bureau of Economic Research, Inc February 2004.

Cooper, Russell, John Haltiwanger, y Laura Power, "Machine Replacement and the Business Cycle: Lumps and Bumps," American Economic Review, September 1999, 89 (4), 921-946.

Cooper, Russell W. and John C. Haltiwanger, "On the Nature of Capital Adjustment Costs," Review of Economic Studies, 07 2006, 73 (3), 611-633. 
Doms, Mark E. and Timothy Dunne, "Capital Adjustment Patterns in Manufacturing Plants," Review of Economic Dynamics, April 1998, 1 (2), 409-429.

Ejarque, Joao and Oivind Anti Nilsen, "Identifying Adjustment Costs of Net and Gross Employment Changes," IZA Discussion Papers 3703, Institute for the Study of Labor (IZA) September 2008.

Ejarque, João Miguel and Pedro Portugal, "Labor Adjustment Costs in a Panel of Establishments: A Structural Approach," IZA Discussion Papers 3091, Institute for the Study of Labor (IZA) October 2007.

Fraumeni, Barbara M., "The measurement of depreciation in the U.S. national income and product accounts," Survey of Current Business, July 1997, 77, 7-42.

Gourio, François, "Estimating Firm-Level Risk," Working Paper, Boston University 2008.

Huggett, Mark and Sandra Ospina, "Does productivity growth fall after the adoption of new technology?," Journal of Monetary Economics, August 2001, 48 (1), 173-195.

Lee, Bong-Soo and Beth Fisher Ingram, "Simulation estimation of time-series models," Journal of Econometrics, February 1991, 47 (2-3), 197-205.

McFadden, Daniel, "A Method of Simulated Moments for Estimation of Discrete Response Models without Numerical Integration," Econometrica, September 1989, $57(5), 995-1026$.

Nilsen, Oivind Anti and Fabio Schiantarelli, "Zeros and Lumps in Investment: Empirical Evidence on Irreversibilities and Nonconvexities," The Review of Economics and Statistics, December 2003, 85 (4), 1021-1037.

Olley, S. and A. Pakes, "The Dynamics of Productivity in the Telecommunications Equipment Industry," Econometrica, 11 1996, 64 (6), 1263-1297.

Pakes, Ariel and David Pollard, "Simulation and the Asymptotics of Optimization Estimators," Econometrica, September 1989, 57 (5), 1027-57.

Pavcnik, Nina, "Trade Liberalization, Exit, and Productivity Improvements: Evidence from Chilean Plants," NBER Working Papers 7852, National Bureau of Economic Research, Inc August 2000. 
Peluffo, Cecilia, "Costos de movilidad intersectorial del empleo, shocks comerciales y tecnológicos: Resultados para Argentina en base a un modelo estructural," Department of Economics, Journal, January-D 2010, 0, 105-144.

Rust, John, "Numerical dynamic programming in economics," in H. M. Amman, D. A. Kendrick, y J. Rust, eds., Handbook of Computational Economics, Vol. 1 of Handbook of Computational Economics, Elsevier, June 1996, chapter 14, pp. 619-729.

Tauchen, George and Robert Hussey, "Quadrature-Based Methods for Obtaining Approximate Solutions to Nonlinear Asset Pricing Models," Econometrica, March 1991, 59 (2), 371-96. 


\section{Tablas y Gráficos}

Tabla 1: EIA: Estadísticas Descriptivas

\begin{tabular}{lccccc}
\hline Variables & $\begin{array}{c}\text { Nro. de } \\
\text { Obs. }\end{array}$ & $\begin{array}{c}\text { Nro. de } \\
\text { Plantas }\end{array}$ & Media & $\begin{array}{c}\text { Desvío } \\
\text { Estándar }\end{array}$ & Mediana \\
\hline Valor Bruto de Producción & 4544 & 568 & 29131.463 & 95944.245 & 6024.071 \\
Valor Agregado & 4544 & 568 & 11052.612 & 43687.595 & 2128.465 \\
Capital & 4504 & 568 & 15032.341 & 69858.899 & 2050.328 \\
Inversión & 4544 & 568 & 999.468 & 11899.926 & 71.2160 \\
Materiales & 4540 & 568 & 18307.990 & 61856.211 & 3410.939 \\
Número de Empleados & 4525 & 568 & 126.531 & 292.779 & 57 \\
\hline
\end{tabular}

Notas: Todas las variables, excepto el número de empleados, son medidas en miles de pesos constantes de 1993. Número de empleados es medido en términos de la cantidad de obreros afectados al proceso productivo.

Tabla 2: Momentos - Tasa de inversión $(i)$

\begin{tabular}{lccccc}
\hline & \multicolumn{5}{c}{ Período } \\
Momentos & $\mathbf{1 9 9 4 - 2 0 0 1}$ & $\mathbf{1 9 9 4}$ & $\mathbf{1 9 9 6}$ & $\mathbf{1 9 9 8}$ & $\mathbf{2 0 0 1}$ \\
\hline media $(i)$ & 0.0971 & 0.1428 & 0.1026 & 0.1176 & 0.0423 \\
sd $(i)$ & 0.2289 & 0.3153 & 0.1690 & 0.2433 & 0.1953 \\
asimetria $(i)$ & 6.8621 & 7.6023 & 2.8465 & 6.4113 & -0.5055 \\
$\operatorname{prop}(|i|<0.01)$ & 0.2917 & 0.2149 & 0.2504 & 0.2522 & 0.4387 \\
$\operatorname{corr}(i, i,-1)$ & 0.1870 & - & - & - & - \\
$\operatorname{prop}(i>0.20)$ & 0.1357 & 0.1865 & 0.1439 & 0.1652 & 0.0657 \\
$\operatorname{prop}(i<-0.05)$ & 0.0271 & 0.0142 & 0.0160 & 0.0142 & 0.0533 \\
$\operatorname{prop}(i<-0.01)$ & 0.0448 & 0.0249 & 0.0302 & 0.0355 & 0.0728 \\
$\operatorname{corr}(i, a)$ & 0.1803 & 0.1696 & 0.1647 & 0.1214 & 0.2011 \\
\hline
\end{tabular}

Notas: media(i): media de la tasa de inversión; sd(i): desvío estándar de la tasa de inversión; asimetria $(i)$ : coeficiente de asimetría de la tasa de inversión; $\operatorname{prop}(|i|<0.01)$ : inacción; $\operatorname{corr}\left(i, i_{-1}\right)$ : correlación serial de la tasa de inversión; $\operatorname{prop}(i>0.20)$ : fracción de observaciones con tasa de inversión superior al $20 \%$; $\operatorname{prop}(i<-0.05)$ : fracción de observaciones con tasa de inversión inferior al $-5 \%$; $\operatorname{prop}(i<-0.01)$ : fracción de observaciones con tasa de inversión negativa; $\operatorname{corr}(i, a)$ : correlación entre la tasa de inversión y shocks de rentabilidad. 
Tabla 3: Estimaciones - Función de Producción

\begin{tabular}{lccc}
\hline & $\begin{array}{c}\text { Mínimos } \\
\text { Cuadrados } \\
\text { Variables }\end{array}$ & $\begin{array}{c}\text { Efectos } \\
\text { Ordinarios }\end{array}$ & Olley-Pakes \\
\hline Trabajo & 0.2023 & 0.2898 & 0.2171 \\
& $(0.0065)$ & $(0.0120)$ & $(0.0108)$ \\
Materiales & 0.7092 & 0.5522 & 0.6757 \\
& $(0.0053)$ & $(0.0089)$ & $(0.0083)$ \\
Capital & 0.0931 & 0.0393 & 0.0910 \\
& $(0.0048)$ & $(0.0164)$ & $(0.0616)$ \\
\hline Observaciones & 4489 & 4489 & 3353 \\
Plantas & 563 & 563 & 541 \\
\hline
\end{tabular}

Notas: Variables expresadas en logaritmos. La variable dependiente es el logaritmo natural del valor bruto de producción (en términos reales).

Errores estándar entre paréntesis. En el caso de las estimaciones Olley-Pakes, los errores estándar fueron computados mediante bootstrap.

Tabla 4: Parámetros

\begin{tabular}{lccc}
\hline Categoría & Símbolo & Definición & $\begin{array}{c}\text { Valor del } \\
\text { parámetro }\end{array}$ \\
\hline & $\alpha_{l}$ & Participación del trabajo en el ingreso & 0.2171 \\
& $\alpha_{m}$ & Participación de materiales en el ingreso & 0.6757 \\
Producción y Demanda & $\alpha_{k}$ & Participación del capital en el ingreso & 0.0910 \\
& $\delta$ & Tasa de depreciación del capital & 0.0991 \\
& $\varepsilon$ & Elasticidad de demanda de mercado & 5.2843 \\
\hline Proceso Estocástico & & & 0.8830 \\
& $\rho_{e}$ & Autocorrelación shock idiosincrásico & 0.6991 \\
& $\sigma_{e}$ & Desvío innovaciones shock idiosincrásico & \\
\hline
\end{tabular}

Tabla 5: Parámetros de costos estimados

\begin{tabular}{|c|c|c|c|c|c|}
\hline \multicolumn{6}{|c|}{ Parámetros } \\
\hline \multicolumn{2}{|c|}{$f$} & \multicolumn{2}{|c|}{$\gamma$} & \multicolumn{2}{|l|}{$p_{s}$} \\
\hline \multicolumn{2}{|c|}{$\begin{array}{c}0.1001 \\
(0.0403)\end{array}$} & \multicolumn{2}{|c|}{$\begin{array}{c}0.0315 \\
(0.0105)\end{array}$} & \multicolumn{2}{|l|}{$\begin{array}{c}0.8836 \\
(0.0727)\end{array}$} \\
\hline \multicolumn{6}{|c|}{ Momentos } \\
\hline & $\operatorname{corr}\left(i, i_{-1}\right)$ & $\operatorname{corr}(i, a)$ & $\operatorname{prop}(i>20 \%)$ & $\operatorname{prop}(i<-5 \%)$ & $\Gamma\left(\widehat{\Theta_{K}}\right)$ \\
\hline Observados & 0.187 & 0.180 & 0.136 & 0.027 & - \\
\hline Simulados & 0.087 & 0.290 & 0.139 & 0.025 & 43.293 \\
\hline
\end{tabular}

Nota: Estimaciones realizadas mediante el método de momentos simulados (MMS).

Errores estándar entre paréntesis. 
Tasa de Inversión

Industria Manufacturera Arg. (1994-2001)

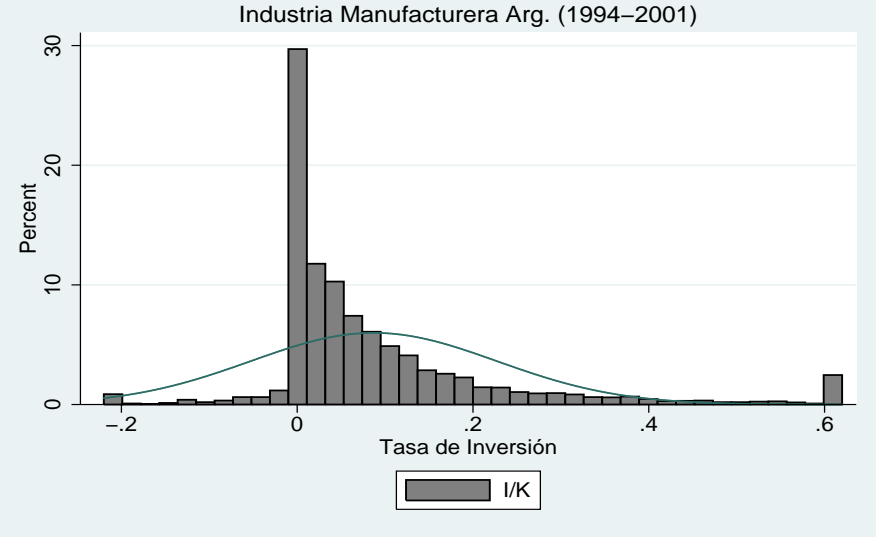

Gráfico 1: Histograma de la tasa de inversión. Elaboración propia en base a datos de la Encuesta Industrial Anual.

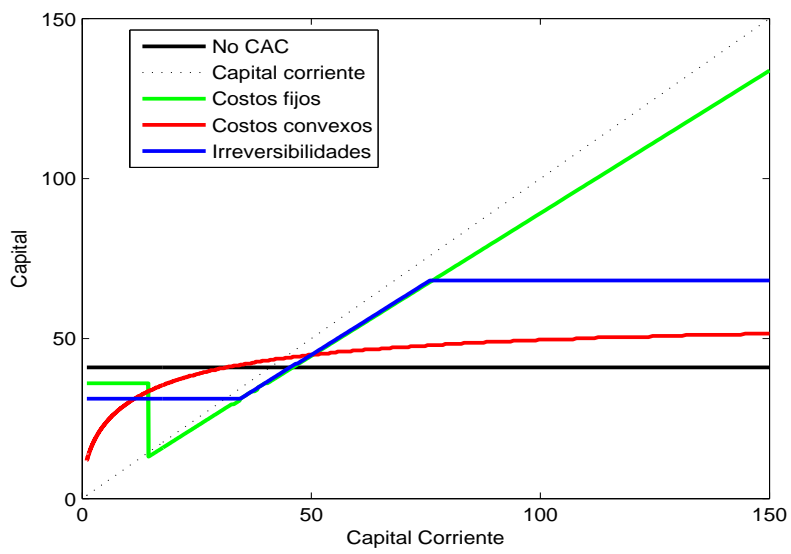

Gráfico 2: Reglas de decisión óptimas: modelos restringidos. El gráfico presenta las reglas de decisión óptimas para diferentes versiones restringidas del modelo presentado en la sección 2. En particular, se exhiben las reglas de decisión correspondientes al modelo con sólo costos cuadráticos $\left(\gamma=0.0315 ; f=0 ; p_{s}=1\right)$; sólo costos fijos $\left(\gamma=0 ; f=0.1001 ; p_{s}=1\right)$; sólo irreversibilidades $\left(\gamma=0 ; f=0 ; p_{s}=0.8836\right)$; y el modelo sin fricciones $\left(\gamma=0 ; f=0 ; p_{s}=1\right)$. 


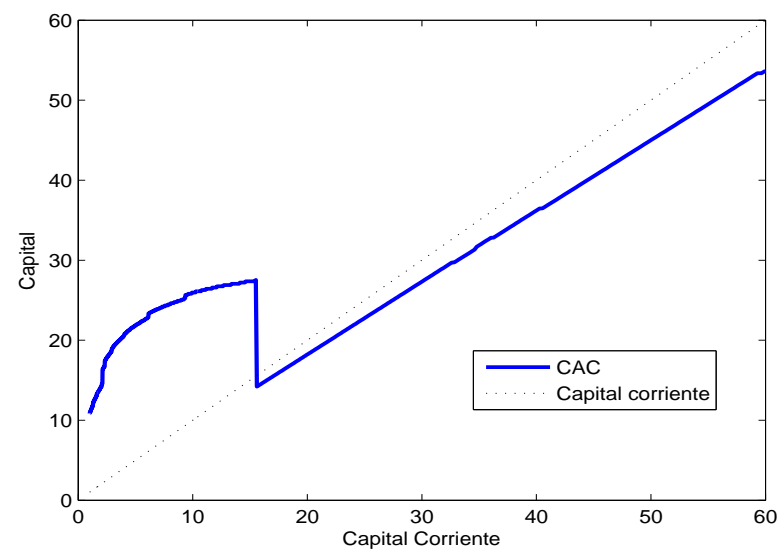

Gráfico 3: Reglas de decisión óptimas: modelo irrestricto. El gráfico presenta la regla de decisión óptima para el modelo estimado. La misma es obtenida para un estado de rentabilidad dado (arbitrario) y en base al vector estimado de parámetros de costos de ajuste del capital $\left(\gamma=0.0315 f=0.1001 ; p_{s}=0.8836\right)$.

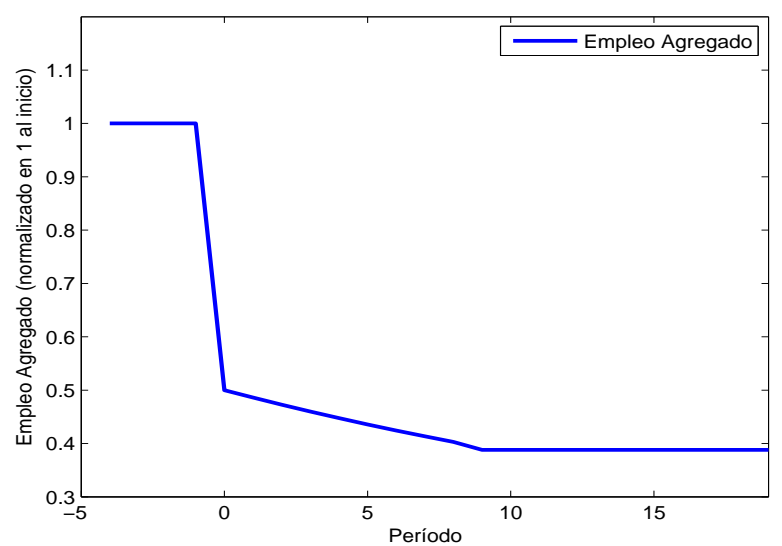

Gráfico 4: Evolución del empleo agregado. Oferta de trabajo perfectamente elástica. El gráfico presenta la dinámica seguida por el empleo agregado luego de una reducción en la rentabilidad media de las firmas del $50 \%$ en el período 0 . El nivel agregado de empleo es normalizado en 1 al inicio. 


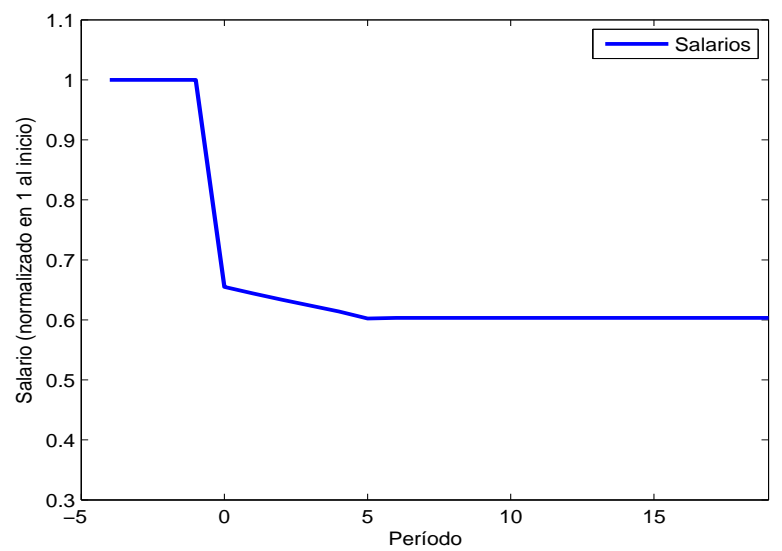

Gráfico 5: Evolución del salario. Oferta de trabajo fija. El gráfico presenta la dinámica seguida por el salario luego de una reducción en la rentabilidad media de las firmas del $50 \%$ en el período 0 . El salario es normalizado en 1 al inicio.

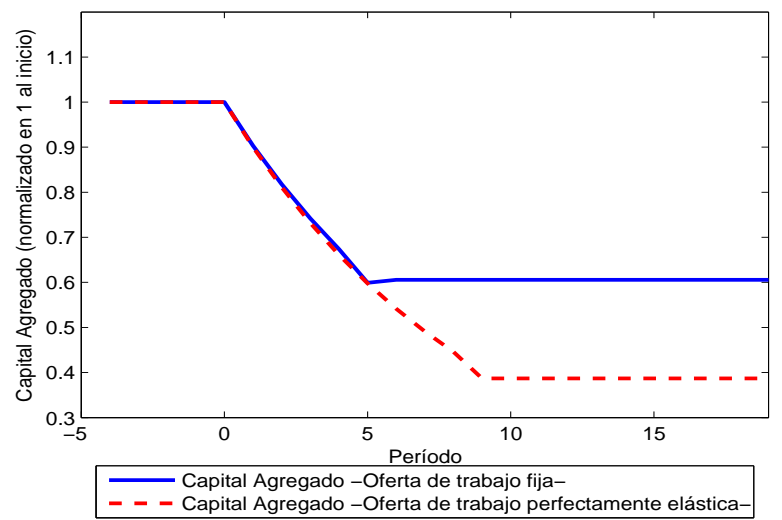

Gráfico 6: Evolución del capital agregado. El gráfico presenta la dinámica seguida por el capital agregado luego de una reducción en la rentabilidad media de las firmas del $50 \%$ en el período 0 . El nivel de capital agregado es normalizado en 1 al inicio. 


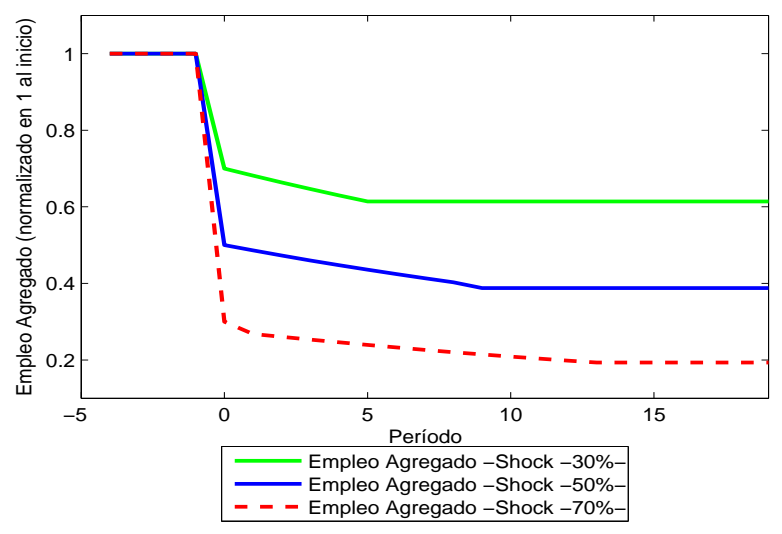

Gráfico 7: Evolución del empleo agregado bajo distintas magnitudes del shock. El gráfico presenta la dinámica seguida por el empleo agregado bajo distintas reducciones en la rentabilidad media de las firmas en el período 0. La oferta de trabajo es perfectamente elástica. El nivel agregado de empleo es normalizado en 1 al inicio.

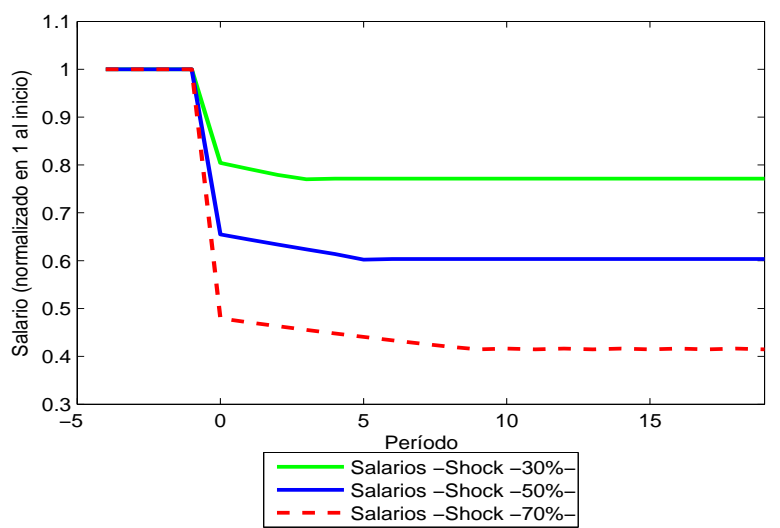

Gráfico 8: Evolución del salario bajo distintas magnitudes del shock. El gráfico presenta la dinámica seguida por el salario bajo distintas reducciones en la rentabilidad media de las firmas en el período 0. La oferta de trabajo está fija. El salario es normalizado en 1 al inicio. 


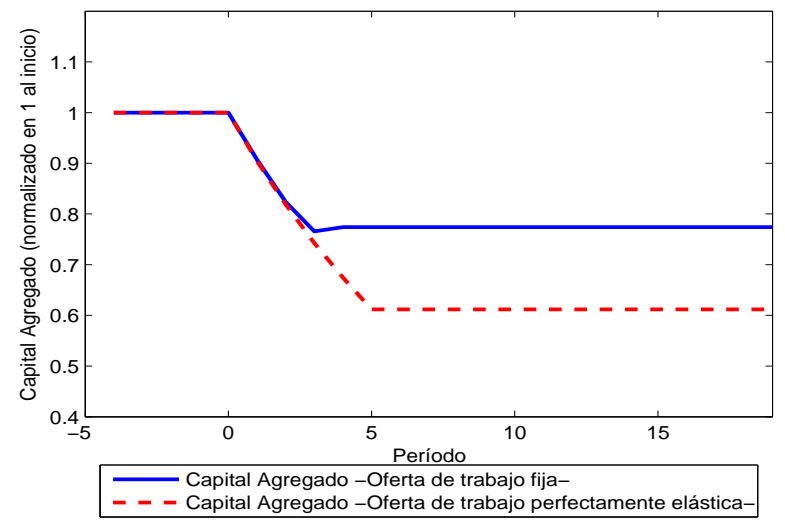

Gráfico 9: Evolución del capital agregado. Reducción en la rentabilidad media del $30 \%$. El gráfico presenta la dinámica seguida por el capital agregado luego de una reducción en la rentabilidad media de las firmas del $30 \%$ en el período 0. El nivel de capital agregado es normalizado en 1 al inicio.

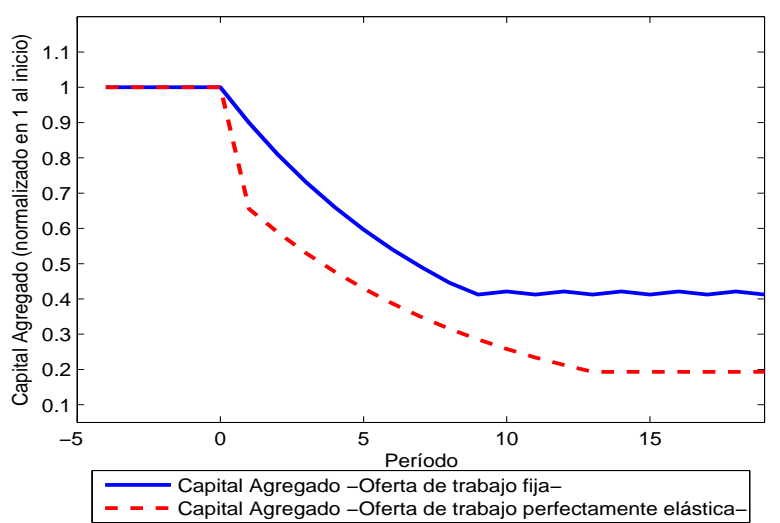

Gráfico 10: Evolución del capital agregado. Reducción en la rentabilidad media del $\mathbf{7 0} \%$. El gráfico presenta la dinámica seguida por el capital agregado luego de una reducción en la rentabilidad media de las firmas del $70 \%$ en el período 0 . El nivel de capital agregado es normalizado en 1 al inicio. 


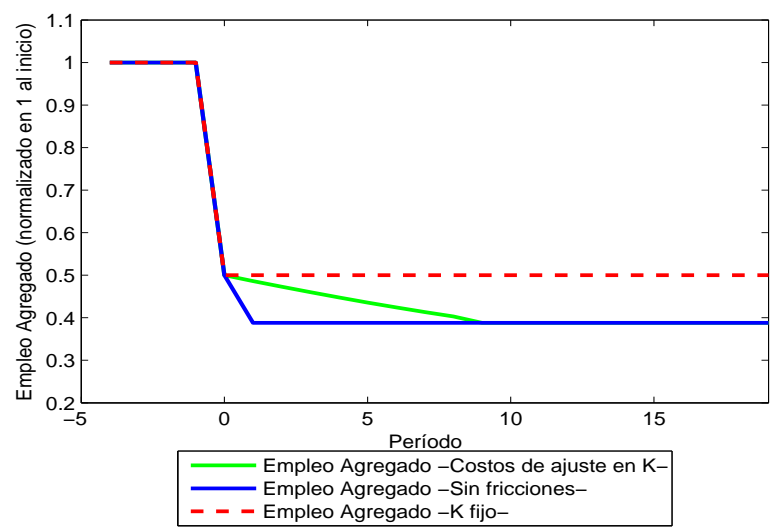

Gráfico 11: Evolución del empleo agregado bajo distintos supuestos de movilidad del capital. El gráfico presenta la dinámica seguida por el empleo agregado bajo distintos supuestos de movilidad del capital y para una reducción del $50 \%$ en la rentabilidad media de las firmas en el período 0. La oferta de trabajo es perfectamente elástica. El nivel agregado de empleo es normalizado en 1 al inicio.

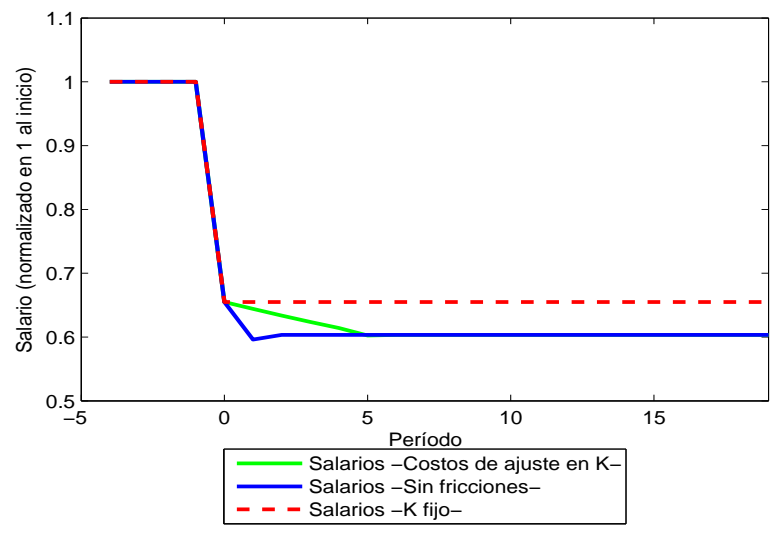

Gráfico 12: Evolución del salario bajo distintos supuestos de movilidad del capital. El gráfico presenta la dinámica seguida por el salario bajo distintos supuestos de movilidad del capital y para una reducción del $50 \%$ en la rentabilidad media de las firmas en el período 0 . La oferta de trabajo está fija. El salario es normalizado en 1 al inicio. 


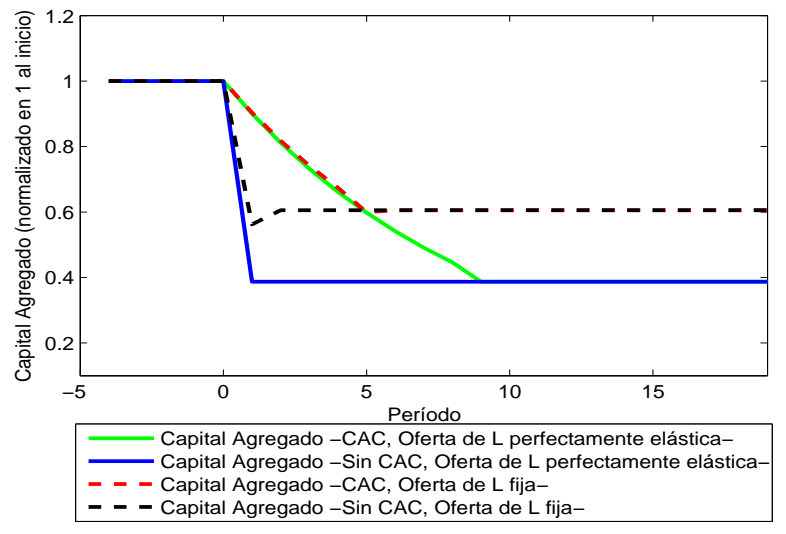

Gráfico 13: Evolución del capital agregado bajo distintos supuestos de movilidad del capital y de comportamiento del mercado de trabajo. El gráfico presenta la dinámica seguida por el capital agregado, bajo distintos supuestos de funcionamiento del mercado de trabajo y de movilidad del capital, luego de una reducción en la rentabilidad media de las firmas del $50 \%$ en el período 0 . El nivel de capital agregado es normalizado en 1 al inicio. 